\title{
Beiträge zur Kenntnis der Cuproverbindungen I.
}

\author{
Von \\ G. BODLÄNDER und O. STORBECK.
}

Mit 3 Figuren im Text.

Die chemischen Eigenschaften der Metalle werden bedingt einerseits durch ihre Stellung im periodischen System, andererseits aber auch durch ihre Stellung in der Spannungsreihe, d. h. durch die Spannung, die nötig ist, um die Elemente - insbesondere die Metalle - aus gleich konzentrierten Lösungen ihrer normalen Salze abzuscheiden.

Diese als Haftintensität bezeichnete Gröfse fällt zum Teil mit der Elektroaffinität ${ }^{1}$ zusammen; ihr Wert bestimmt die Widerstandsfähigkeit der freien Metalle gegen Atmosphärilien, ihr Verhalten gegen Säuren und ihre Fähigkeit, sich gegenseitig zu verdrängen, während die Stellung im periodischen System vor allem die Wertigkeit und damit den Typus der Verbindungen bestimmt.

Die Haftintensität steht auch in naher Beziehung zu der Löslichkeit der Metallverbindungen und zu ihrer Fähigkeit, komplexe Verbindungen zu bilden. Es ist deshalb mit Freude zu begrüfsen, dafs die Haftintensitäten der meisten Metalle jetzt mit ziemlicher Genauigkeit festgestellt ${ }^{2}$ sind. Nur für die Metalle, die fast nur in Form komplexer Ionen in Lösungen enthalten sind, wie z. B. Gold, Platin, Antimon, Arsen fehlen genaue Angaben.

Es kann immer nur direkt die Haftintensität eines Metalles gegen solche Lösungen bestimmt werden, die neben dem Metall existenzfähig sind; so lälst sich die Haftintensität des Eisens direkt

1 Abegg und Bodländer, Die Elektroaffinität ein neues Prinzip der chemischen Systematik. Z. anorg. Chem. 20 (1899), 453-499.

2 Wirsmore, Über Elektrodenpotentiale. Zeitschr. phys. Chem. 35, (1900), 291.

Z. anorg. Chem. XXXI. 
nur gegen Ferrosalzlösungen bestimmen, weil Ferrisalz in Berührung mit dem Metall in Ferrosalz übergeht. Es kann daher durch direkte Messung nur die Spannung ermittelt werden, welche man aufwenden mufs, um die zweiwertigen Ionen des Eisens in metallisches Eisen überzuführen. Dagegen ist es nicht möglich, direkt festzustellen, welche Spannung man braucht, um den dreiwertigen Ferriionen ihre Ladung zu entziehen.

Wenn man Beziehungen aufsucht zwischen der Haftintensität und den Eigenschaften der Verbindungen, so gelten diese nur für die Ferrosalze; nur auf indirektem Wege ist es möglich, auch die Spannung zu bestimmen, welche Eisen gegen eine Ferrisalzlösung haben würde.

Ähnliches liegt beim Quecksilber vor. ${ }^{1}$ Neben dem Metall sind fast nur Lösungen von Mercurosalzen existenzfähig, die Haftintensität des Quecksilbers bezieht sich also nur auf den Übergang von Quecksilber in Mercuroionen und umgekehrt. Nur für die Eigenschaften der Mercuroverbindungen lassen sich Beziehungen aus der Haftintensität ableiten; die Eigenschaften der von den Mercuro- gänzlich verschiedenen Mercuriverbindungen lassen sich nur aus der indirekt bestimmbaren Spannung ableiten, die beim Übergang von Quecksilber in Mercuriionen aufgewandt wird.

Kupfer ist in Berührung mit dem Metall meist nur in Form der Cuprosalze beständig, wenigstens so weit Lösungen von grölserer Konzentration der Metallionen in Betracht kommen. Es ist daher auch nur die Spannung bekannt, die beim Übergang des Metalls in Cupriionen aufzuwenden ist. Es ist allerdings leicht, die Spannung zu messen, welche das Metall gegen die Lösung eines Cuprosalzes z. B. von Cuprochlorid in Chlorkalium zeigt. Für den Vergleich der Eigenschaften verschiedener Metalle können aber nur diejenigen Spannungen in Betracht kommen, die die Metalle gegen ihre Lösungen zeigen, welche gleiche Konzentrationen der freien Metallionen besitzen.

Als Haftintensität bezeichnet man gewöhnlich die Spannung. welche eine Lösung von normaler Konzentration • der Metallionen gegen das Metall aufweist. Ist die Konzentration der Metallionen grölser, so wird diese Spannung verringert, ist sie kleiner, vermehrt.

1 Vergl.: OGG, Über das chemische Gleichgewicht zwischen Amalgamen und Lösungen. Zeitschr. phys. Chem. 27 (1898), 285-311.

Aвrx, Über das Gleichgewicht zwischen den verschiedenen Oxydationsstufen desselben Metalles. Z. anorg. Chem. 26 (1901), 361-437. 
Kennt man die Konzentration der Metallionen, so kann man aus der beobachteten Spannung die der normalen Lösung berechnen. Für die Cuprosalze waren aber Lösungen von bekanntem Gehalt an Cuproionen noch nicht untersucht worden; es war daher die Haftintensität des Kupfers bei dem Übergang in Cuproionen noch nicht ermittelt. Die Kenntnis dieser Gröfse ist schon deshalb von Wichtigkeit, weil sie die Prüfung der Frage gestattet, ob die von den Eigenschaften der Cuprisalze gänzlich verschiedenen Eigenschaften der Cuprosalze sich aus der Haftintensität der Cuproionen ableiten lassen. Es lag daher in unserer Absicht, die Entladungsspannung der Cuproionen in Lösungen bekannter Ionenkonzentration zu bestimmen. Die Aufgabe wurde durch das Auftauchen zahlreicher Vor- und Nebenfragen im Laufe der Untersuchung erschwert. Während wir mit ihr beschäftigt waren, sind Arbeiten über denselben oder ähnliche Gegenstände, insbesondere von ABEI ${ }^{1}$ und von LUTHER ${ }^{2}$ erschienen. Wir werden auf diese Arbeiten, deren Ergebnisse nur teilweise durch die unseren bestätigt werden, später zurückkommen.

Die einfachen Cuproverbindungen gelten als in Wasser ,unlöslich". Soweit sie, wie z. B. Kupfercyanür oder Kupferchlorür, durch Zuführung von Neutralsalzen oder von Ammoniak in Lösung gebracht werden können, sind sie in diesen Lösungen in Form komplexer Salze bezw. komplexer Ionen enthalten.

Die Möglichkeit der Bestimmung der Cuproionen bot nur die Untersuchung der rein wässerigen Lösung von einfachen Cuproverbindungen.

Es war nach der Elektroaffinität des Chlors zu erwarten, dafs von den Cuprohaloiden das Cuprochlorid am leichtesten löslich sein würde. BODLÄNDER ${ }^{3}$ berechnet die Löslichkeit des Cuprochlorids angenähert zu $8.5 \cdot 10^{-3}$ - es müfste also 1 Liter $0.85 \mathrm{~g}$ Cuprochlorid enthalten.

Wenn auch die wichtigste Grundlage der Berechnurg, gerade die Haftintensität, fehlte, so war doch anzunehmen, dafs die Löslichkeit etwa von dieser Gröfsenordnung sein würde und somit analytisch leicht ermittelt werden konnte.

1 I. c.

a Über das elektromotorische Verhalten von Stoffen mit mehreren Oxydationsstufen. Zeitschr. phys. Chem. 36 (1901), 385.

3 BoDländer, Über Beziehungen zwischen Löslichkeit und Bildungawärme von Elektrolyten. Zeitschr. phys. Chem. 27 (1898), 55. 
Bei Ausschlufs jeder Oxydation durch den Luftsauerstoff sollte deshalb die Löslichkeit des Cuprochlorids festgestellt werden.

Es ergab sich bei diesen Versuchen, dafs das Cuprochlorid nicht unzersetzt in Lösung geht. Das rein weifse Salz färbte sich bei Behandlung mit reinem Wasser rot. Das konnte herrühren von einer hydrolytischen Spaltung des Salzes in Kupferhydroxydul und Salzsäure. Die Farbe und Beschaffenheit des roten Niederschlages war aber von der des Kupferhydroxyduls verschieden. Dieses selbst trat auch in geringen Mengen als orangegelbe Trübung auf. Die Hauptmenge des dem Cuprochlorid beigemengten Niederschlages hatte aber rein kupferrote Farbe. Grölsere Partikeln, die sich bei längerem Stehen der sauerstofffreien Lösung in Berührung mit dem Cuprochlorid bildeten, hatten deutlich metallischen Glanz; das wies darauf hin, dafs neben der in geringem Umfange stattfindenden hydrolytischen Spaltung:

$$
\mathrm{CuCl}+\mathrm{H}_{2} \mathrm{O}=\mathrm{Cu}(\mathrm{OH})+\mathrm{HCl}
$$

auch ein Zerfall nach der Gleichung stattfindet:

$$
2 \mathrm{CuCl}=\mathrm{CuCl}_{2}+\mathrm{Cu} \text {. }
$$

Die erste Zersetzung des Cuprochlorids durch Wasser war von LESCOEUR ${ }^{1}$ und von J. K. HAYwOOD ${ }^{2}$ beobachtet worden, die zweite war bisher nicht bekannt; sie ist analog der Abscheidung von metallischem Kupfer aus cuprosulfathaltigen Lösungen von Cuprisulfat, welche Fönster ${ }^{3}$ beobachtet hat. ${ }^{4}$ Der Kupfergehalt der Lösungen von Cuprochlorid in Wasser ist so grofs, dafs er analytisch leicht bestimmt werden konnte.

Die Lösungen enthalten aber neben dem Cuprochlorid, wie sich aus den Umsetzungsgleichungen ergiebt, auch Salzsäure und Kupferchlorid, das letztere in überwiegender Menge. Es kam also darauf

1 Ann. Chim. phys. [7] 2 (1894), 97.

2 Journ. phys. Chem. 1 (1897), 411.

${ }^{3} Z$. anorg. Chem. 14 (1896), 119.

${ }^{4}$ Die gleiche Zersetzung des Kupferchlorïrs in Kupfer und Kupferchlorid hat Gröger (Z. anorg. Chem. 28 (1901), 156) beobachtet. $\mathrm{Er}$ hat offenbar übersehen, dals wir über diese Reaktion schon im September 1900 auf der Naturforscherversammlung in Aachen berichtet haben. (Vergl. Chem. Ztg. 24 (19(10), 82; Zeitschr. Eleltrochem. 7 (1900), 159.) 
an, auch die beiden Verbindungsstufen des Kupfers getrennt zu ermitteln.

Das Kupfer des Cuprochlorids war nicht vollständig in Form von Cuproionen vorhanden, auf deren Bestimmung es allein ankam. Die Konzentration des Cuprochlorids war, wenn auch klein, doch immer so grofs, dafs man seine elektrolytische Dissoziation nicht als vollständig ansehen konnte.

Noch mehr zu berücksichtigen war, dafs ein Teil des Cuprochlorids in Form komplexer Ionen vorhanden sein mufste. Es ist bekannt, dafs Cuprochlorid sich in Salzsäure und Chloriden ziemlich leicht löst. Die Löslichkeit in Salzsäure ist von Le Ceaterier ${ }^{\text {I }}$ und von EvGEL ${ }^{2}$ sowie von $\mathrm{ABEL}^{3}$ bestimmt worden, sie steigt bei zunehmendem Salzsäuregehalt sehr rasch an. Es war anzunehmen, dals das Cuprochlorid in diesen Lösungen in Form komplexer Anionen $(\mathrm{CuCl})_{m} . \mathrm{Cl}_{n}$ vorhanden ist. Die Menge des in dieser Form gelösten Kupfers kann nur von der Menge der Chlorionen abhängen. Es mufsten sich also solche Ionen auch bilden durch die Einwirkung der Chlorionen des Cuprichlorids und der Chlorionen des freien dissoziierten Cuprochlorids. Es war daher nötig, durch besondere Versuche die Abhängigkeit der Menge der komplexen Ionen von der der Chlorionen festzustellen. Wenn die komplexen Ionen die Formel $\mathrm{CuCl}_{2}^{\prime}$ besafsen, so war nach der Gleichung:

$$
\begin{gathered}
\mathrm{CuCl}+\mathrm{Cl}^{\prime}=\mathrm{CuCl}_{2}^{\prime} \\
\frac{\mathrm{CuCl} \cdot \mathrm{Cl}^{\prime}}{\mathrm{CuCl}_{2}^{\prime}}=k
\end{gathered}
$$

zu erwarten, dafs die Menge der komplexen Ionen bei Gegenwart von Cuprochlorid als Bodenkörper der Menge der freien Chlorionen proportional sein würde.

Gleiches gilt aber auch, wenn das Cuprochlorid die Formel $\mathrm{Cu}_{2} \mathrm{Cl}_{2}$ oder $\mathrm{Cu}_{3} \mathrm{Cl}_{3}$ oder $\mathrm{Cu}_{n} \mathrm{Cl}_{n}$ besitzt, die komplexen Ionen aber durch Anlagerung von einem Chlorion an das neutrale Salz gebildet werden. Auch dann ist, wenn Cuprochlorid Bodenkörper ist, nach den Gleichungen:

$$
\mathrm{Cu}_{n} \mathrm{Cl}_{n}+\mathrm{Cl}^{\prime}=\mathrm{Cu}_{n} \mathrm{Cl}_{n}{ }^{\prime}+1
$$

1 Compt. rend. 98 (1884), 814.

2 Ann. Chim. Phys. [6] 17 (1889), 376.

s. c. 


$$
\begin{gathered}
6- \\
\frac{\mathrm{Cu}_{n} \mathrm{Cl}_{n} \cdot \mathrm{Cl}^{\prime}}{\mathrm{Cu}_{n} \mathrm{CI}_{n}{ }^{\prime}+1}=k
\end{gathered}
$$

die Menge der komplexen Ionen der Menge der freien Chlorionen proportional.

Wenn aber sich ein Molekỉl des Cuprochlorids mit zwei Ionen Chlor zu komplexen Ionen verbindet, so ist nach der Gleichung:

$$
\begin{gathered}
\mathrm{CuCl}+2 \mathrm{Cl}^{\prime}=\mathrm{CuCl}_{3}{ }^{\prime \prime} \\
\frac{\mathrm{CuCl} \cdot\left(\mathrm{Cl}^{\prime}\right)^{2}}{\mathrm{CuCl}_{3}{ }^{\prime \prime}}=k
\end{gathered}
$$

die Menge der komplexen Ionen dem Quadrat der Menge des freien Chlorionen proportional. Auch hier gilt dieselbe Beziehung, auch wenn dem Cuprochlorid die Formel $\mathrm{Cu}_{n} \mathrm{Cl}_{n}$, den komplexen Ionen also die Formel $\mathrm{Cu}_{n} \mathrm{Cl}_{(n+2)}$ zukommt.

Verbindet sich ein Molekül Cuprochlorid mit drei Chlorionen, so ist die Menge der komplexen Ionen der dritten Potenz der Chlorionen proportional.

Nach den Versuchen von Evaer schien die Menge des Cuprochlorids dem Quadrat der Chlorionen proportional anzusteigen; es war zu prüfen, ob dieselbe Beziehung auch für die verdünnten Lösungen gilt, die hier allein in Betracht kommen, oder ob nicht in ihnen Moleküle $\mathrm{Cu}_{n} \mathrm{Cl}_{n+1}$ vorhanden sind.

Für die Entscheidung der Frage, auf die es uns hauptsächlich ankam, war es ferner von Wichtigkeit zu wissen, ob die freien Cuproionen ein- oder zweiatomig sind, ob ihnen die Formel:

$$
\mathrm{Cu} \cdot \text { oder } \mathrm{Cu}_{2} \cdot \text { zukommt. }
$$

Die Löslichkeit des Cuprochlorids ist ja nicht so grols, dals man direkt die Haftintensität der Cuproionen in normalen Lösungen bestimmen konnte. Bestimmt man sie aber bei geringerer Konzentration, so war es für die Umrechnung auf normale Konzentration nötig, die Anzahl der Ladungen zu kennen, welche jedes Ion trägt.

Ist die Konzentration eines Ions $p$-normal und seine Entladungsspannung bei dieser Konzentration $=e$, so ist seine Haftintensität in normalen Lösungen

$$
E=e+\frac{0.058}{n} \log p .
$$


Für die Berechnung von $E$ aus $e$ ist also wichtig, aufser der Konzentration $p$ auch die Anzahl $n$ der Ladungen jedes Ions festzustellen. Haben die Cuproionen die Formel $\mathrm{Cu}^{\circ}$, so ist $n=1$, haben sie die Formel $\mathrm{Cu}_{2}{ }_{2}$, so ist $n=2$.

Die Lösung dieser für unseren nächsten Zweck sekundären Fragen haben wir in Angriff genommen und fortgeführt, obwohl uns bekannt war, dafs ein Teil von ihnen auch schon im Laboratorium von NERNST durch ABEL bearbeitet wurde.

Es geschah dies, weil vor der Entscheidung dieser Fragen die Lösung der Hauptaufgabe, der Bestimmung der Haftintensität der freien Cuproionen, nicht möglich war. Es sind bei unseren Untersuchungen neue Methoden in Anwendung gekommen, so dals, was der Erfolg bestätigte, von ihnen eine neue Beleuchtung des Gegenstandes erwartet werden konnte.

\section{Darstellung des Cuprochlorids.}

Zur Darstellung des Cuprochlorids wurde Cuprichlorid mit starker Salzsäure und Kupferspänen in einem geräumigen Kolben so lange gekocht, bis die anfangs tief dunkelgrün gefärbte Mischung eine hellgrüne Färbung annahm und das gebildete Cuprochlorid sich auszuscheiden begann. Um das heftige Stofsen beim Kochen möglichst zu vermeiden, verwandten wir lange spiralförmige Kupferstreifen, die bis in den Hals des Kolbens hineinragten; auch wurde die Kolbenöffnung mit einem Trichter bedeckt, um einem zu schnellen Verdampfen der Salzsäure vorzubeugen. Die Mischung wurde von dem Ungelösten in einen bis zur Hälfte mit ausgekochtem Wasser angefüllten Kolben abgegossen, dieser mit einem dreifach durchbohrten Gummistopfen verschlossen und die Flüssigkeit im Wasserstoff- oder Kohlensäurestrom abgekühlt. Nach dem Erkalten schied sich das Cuprochlorid zum gröfsten Teil in prachtroll schneeweifsen Krystallen ab, die durch mehrfaches Auswaschen mit luftfreiem Wasser von dem beigemengten Cuprichlorid und der Salzsäure gereinigt wurden. Nachdem die Salzsäure vollständig ausgewaschen war, trat eine schön rote Färbung auf, die durch die unter Abscheidung von Kupfer erfolgende Zersetzung des Cuprochlorids bedingt war. Ein Beweis dafür, dafs die rote Trübung nicht allein von Kupferoxydul, sondern auch von metallischem Kupfer herrührte, warde dadurch geliefert, dafs sich die 
Trübung in luftfreier konzentrierter Salzsäure nicht klärte, was geschehen wäre, wenn nur Kupferoxydul vorhanden gewesen wäre.

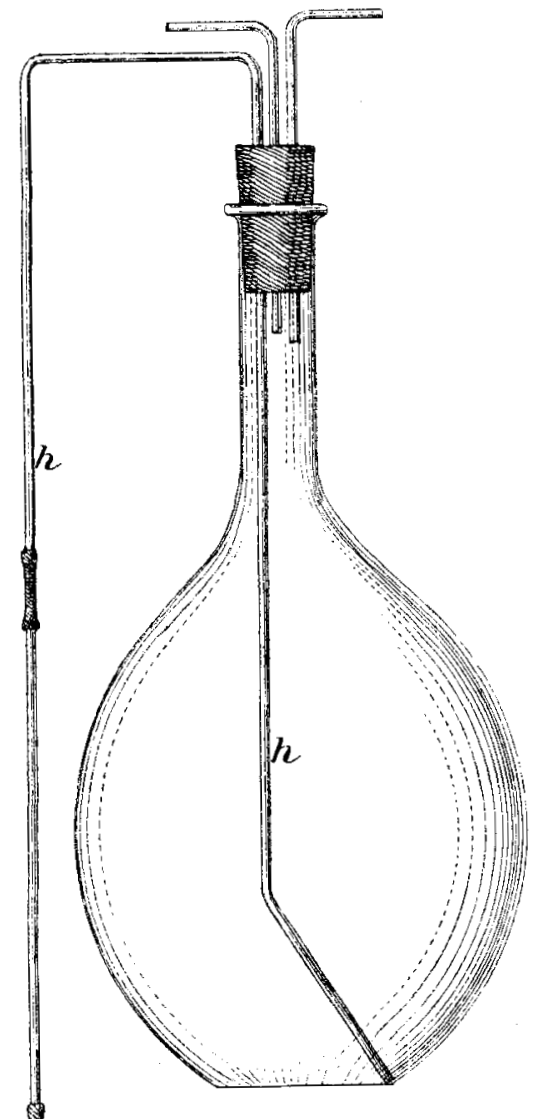

Fig. 1.

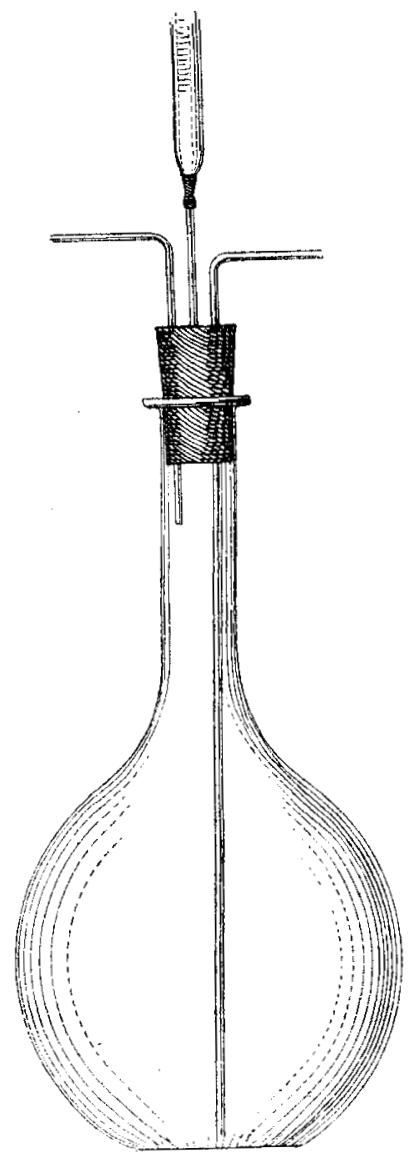

Fig. 2.

f' Erst auf Zusatz von Oxydationsmitteln, z. B. Bichromat, ging das Kupfer in Lösung.

Um während des Auswaschens des Niederschlages und für die Löslichkeitsbestimmungen das Kupferchlorür vor der Oxydation durch den Luftsauerstoff vollständig zu schützen, hatte der etwa zwei Liter fassende Kolben, in welchem sich das Kupferchlorür befand, die Form Figur 1. Durch zwei Öffnungen des dreifach a urchbohrten Stopfens führten kurze, rechtwinklig gebogene Röhren 
zum Zu- und Ableiten des Wasserstoffs oder der Kohlensäure und zum Einfüllen der Flüssigkeiten. In der dritten Bohrung war das zweimal rechtwinklig gebogene Heberrohr $h$ befestigt, durch das die Lösung ziemlich vollständig aus dem Kolben entleert werden konnte. Zum Auswaschen des Niederschlages und zur Herstellung der gesättigten Lösungen diente ausgekochtes und in einer inaktiven Atmosphäre erkaltetes Wasser oder ebenso behandelte Lösungen von Salzen. Die Einfüllung erfolgte, obne dafs die Luft zutreten konnte, unter dem Druck des inaktiven Gases, indem die Flüssigkeit aus dem Kolben, in dem sie ausgekocht und erkaltet war, durch Glasröhren und Schlauchverbindungen in den Kolben 1 gedrückt wurde, während das Gas aus diesem Kolben durch das zweite Rohr unter einem Wasserventil austrat.

Mit dem Heber $h$ war die Filtriervorrichtung $f$ verbunden. Sie bestand aus einem dünnen Glasrohr, das mit einem weiteren, welches sich unten verjüngte und in eine Spitze auslief, verbunden war. Die weitere Glasröhre enthielt in ihrem verjüngten Teil den zum Filtrieren dienenden Wattepfropfen. Der Kolben, Figur 2, welcher das Filtrat aufzunehmen hatte, war ebenfalls mit einem dreifach durchbohrten Stopfen verschlossen; das zum Boden führende Rohr war nur einmal gebogen und das eine kurze Zuleitungsrohr war gerade, damit es mit der Bürette verbunden werden konnte.

Vor der Filtration wurde der Kolben mit sauerstofffreier Kohlensäure gefüllt, das zum Boden führende Rohr mit der Filtriervorrichtung verbunden und das rechtwinklig gebogene mit einer anderen Glasröhre, die in Wasser tauchte, vereinigt, so dafs die Filtration unter Kohlensäuredruck stattfand und ein Luftzutritt in jedem Falle ausgeschlossen wurde. Beim Titrieren mulste natürlich, um ein Zuflielsen aus der Bürette zu ermöglichen, die den Kolben anfüllende Kohlensäure vorsichtig angesogen werden.

Das Cuprichlorid wurde mittels $1 / 20$ Normal-Natriumthiosulfat, deren Titer auf 0.01 Normal-Kupforsulfatlösung wiederholt eingestellt wurde, titriert und zwar in der Weise, dals ca. $10 \mathrm{~g}$ Jodkalium ${ }^{1}$

${ }^{1}$ Ein grolser Überschurs an Jodkalium war für die Titration nötig, weil sonst die Entbindung des dem Cuprikupfer äquivalenten Jods äufserst langsam erfolgt. Der Entbindung des Jods mufs die Abscheidung des Cuprojodids vorangehen; diese wird durch den Überschuls von Jodkalium wegen der Löslichkeitserniedrigung erleichtert. 
in wenig Wasser gelöst und mit wasserlöslicher Stärke verversetzt, ausgekocht und noch heifs unter Vermeidung von Luftzutritt dem Filtrat zugesetzt wurden. Da das Cuprichlorid mit Jodkalium, Cuprojodid, Jod und Chlorkalium bildet nach der Gleichung:

$$
\mathrm{CuCl}_{2}+2 \mathrm{KJ}=\mathrm{CuJ}+2 \mathrm{KCl}+J,
$$

so konnte dasselbe durch die Bindung des freien Jods mittels Natriumthiosulfat leicht bestimmt werden.

Versuche, die freie Salzsäure durch Baryumbydroxyd mittels Methylorange oder Phenolphtalein als Indikator nach der Cuprititrierung in derselben Flüssigkeit zu bestimmen, waren nur möglich, wenn in der Wasserstoffatmosphäre gearbeitet wurde, da die Kohlensäure auf den Indikator reagiert hätte. $\mathrm{Da}$ aber aus Gründen, die später erwähnt werden, die Bestimmung in der Kohlensäureatmosphäre vorzuziehen war, und da ferner die Hydrolyse des Cuprochlorids, die auch schon von Lescoend und Harwood untersucht war, nur untergeordnetes Interesse besal's, so konnte in den meisten Fällen die direkte Bestimmung der Hydrolyse unterlassen werden, zumal deren Betrag sich auch auf indirektem Wege aus den Versuchszahlen ermitteln läfst.

Die Titration von Cuprochlorid mit Kaliumpermanganat unter Zusatz von Schwefelsäure gab keine zuverlässigen Resultate und zumal bei Gegenwart von Chlorkalium. Je nachdem langsam oder schneller titriert wurde, war der Verbrauch von Kaliumpermanganat ein verschiedener, so dals die Annahme wohl gerechtfertigt erscheint, dals der störende Einflufs der Gegenwart von Chloriden zuzuschreiben ist. Es ist möglich, dals das in Form komplexer Verbindungen vorhandene Cuprochlorid durch Permanganat gar nicht oder nur sehr langsam oxydiert wird. Wie die Reduktion des Cuprichlorids durch Kupfer bei Gegenwart von Chloriden erleichtert wird, wird der umgekehrte Prozefs, die Oxydation des Cuprochlorids durch die Gegenwart von viel Chloriden erschwert.

Die Lösung, welche zur Bestimmung des Cuprochlorids gedient hatte, oder eine frische Portion des Filtrates - 1 bis 2 Liter wurde sowohl zur Bestimmung des Chlors auf gewichtsanalytischem Wege als auch zur Bestimmung des Gesamtkupfers nach elektrolytischer Methode verwandt, indem nach Ausfällung des überschüssigen Silbers und Abfiltrieren mit Schwefelsäure eingedampft und nach Zusatz von Salpetersäure elektrolysiert wurde. 
Zur Ausschliefsung des Luftsauerstoffs wurde zuerst in einer Wasserstoffatmosphäre gearbeitet. Gelegentlich der Bestimmung der Leitfähigkeit dieser Lösungen zeigte sich aber, dals aus ihnen Wasserstoff auf platiniertem Platin fast das gesamte Kupfer in kurzer Zeit metallisch niederschlug. Bei Abwesenheit von Platin wurde eine solche Reduktion nicht beobachtet. Es war aber die Möglichkeit nicht ausgeschlossen, dafs eine solche Redultion doch einmal eintreten konnte, und es wurde deshalb meist in einer Kohlensäureatmosphäre gearbeitet. Die Versuche in einer Kohlensäureatmosphäre hatten übrigens dieselben Ergebnisse, wie die unter sonst gleichen Bedingungen angestellten Versuche in der Wasserstoffatmosphäre.

Der Ausschlufs des Luftsauerstoffs war für das Gelingen der Versuche die wichtigste Bedingung. Das konnte nur erreicht werden, wenn der mit bestschlielsenden Stopfen und Schläuchen verschlossene Kolben beständig während der Sättigung und Filtration unter dem Druck eines inaktiven Gases stand, so dafs auch die Diffusion von Sauerstoff in den Kolben ausgeschlossen war. Um das zu erreichen, mufste aber darauf verzichtet werden, die Sättigung bei durchaus konstanter Temperatur in einem Thermostaten vorzunehmen. Vielmehr erfolgte sie durch 6-8stündiges Schütteln bei Zimmertemperatur auf einer Schüttelmaschine, wobei der Kolben durch Glasröhren und Schläuche immer mit dem Kohlensäureapparat.und der Waschvorrichtung verbunden blieb. Die Resultate zeigen, dafs die Schwankungen der Zimmertemperatur die Resultate nicht wesentlich beeinflussen.

Es kam in jedem Falle darauf an, das Gesamtkupfer, das in Form von Cupriverbindungen vorhandene Kupfer, das Chlor, das Cuprokupfer und die freie Salzsäure zu bestimmen.

Die drei ersten Bestimmungen geben scharfe Resultate, namentlich wenn bei der Bestimmung des Cuprikupfers die oben angegebenen Vorsichtsmafsregeln innegehalten wurden.

Sehr unzuverlässig war dagegen die Bestimmung des Cuprokupfers und der freien Salzsäure. Es konnte aber das Cuprokupfer genauer indirekt ermittelt werden, indem das Cuprikupfer von dem Gesamtkupfer abgezogen wurde. Auch die Salzsäure lälst sich

1 Dadurch, dafs sich im Kolben immer fein verteiltes metallisches Kupfer befand und Salzsäure durch Hydrolyse entsteht, werden Spuren eintretenden Sauerstoffs unschädlich gemacht. 
berechnen, wenn man vom Gesamtchlor das an Cupri- und Cuprokupfer gebundene Chlor bei Gegenwart von Chlorkalium auch das daran gebundene abzieht; indessen ist diese Berechnung weniger zuverlässig, weil ihr alle event. Analysenfehler anhaften.

\section{Tabelle 1.}

Löslichkeit des Cuprochlorids in mg-Atomen im Liter.

\begin{tabular}{l|c|c|c|c|c|c}
\hline Temp. & Ges. Kupfer & $\begin{array}{c}\mathrm{CuCl}_{2} \\
\text { mafs- } \\
\text { analytisch }\end{array}$ & Cl & $\begin{array}{c}\mathrm{CuCl} \\
\text { berechnet }\end{array}$ & $\begin{array}{c}\mathrm{CuCl} \\
\text { mals- } \\
\text { analytisch }\end{array}$ & $\begin{array}{c}\mathrm{HCl} \\
\text { berechnet }\end{array}$ \\
\hline \hline
\end{tabular}

a) in Wasserstoffatmosphäre.

\begin{tabular}{r|l|l|l|l|l|l}
- & 2.752 & 2.124 & 5.672 & 0.628 & - & 0.796 \\
20.2 & 2.919 & 2.254 & 5.525 & 0.665 & 0.420 & 0.352 \\
19.6 & 2.971 & 2.294 & 5.464 & 0.677 & 0.474 & 0.199 \\
19.3 & 2.861 & 2.245 & 5.464 & 0.616 & 0.499 & 0.358
\end{tabular}

b) in Kohlensäureatmosphäre.

\begin{tabular}{|c|c|c|c|c|c|c|}
\hline 20.6 & 2.818 & 一 & 5.235 & - & 0.525 & - \\
\hline 21.7 & 2.805 & 2.243 & 5.430 & 0.562 & 0.516 & 0.382 \\
\hline- & 2.880 & 2.258 & 5.312 & 0.662 & 0.391 & 0.274 \\
\hline 19.7 & 2.805 & 2.138 & 5.390 & 0.667 & 0.336 & 0.447 \\
\hline & 2.851 & 2.222 & 5.436 & 0.634 & 0.451 & 0.401 \\
\hline
\end{tabular}

In der vorstehenden Tabelle sind die Bestimmungen der Löslichkeit des Cuprochlorids in reinem Wasser in einer Wasserstoffund einer Kohlensäureatmosphäre zusammengestellt. Wenn auch die Versuchsresultate Schwankungen ergeben, so zeigt sich doch, dafs die Menge des Cuprichlorids und Cuprochlorids annähernd konstant ist.

Es ist diese Konstanz ein weiterer Beweis dafür, dafs das Cuprisalz aus dem Cuprosalz unter Abspaltung von metallischem Kupfer und nicht durch zufällige Oxydation des Cuprochlorids entsteht.

\section{Löslichkeit des Cuprochlorids in Chlorkalium.}

Es ist schon in der Einleitung hervorgehoben worden, dafs keineswegs das gesamte Cuprokupfer der Lösungen in Form freier Cuproionen vorhanden ist und dals ein Teil in Form komplexer Verbindungen mit den Chlorionen zugegen sein mufs. Die Menge der freien Cuproionen konnte nur ermittelt werden, wenn derjenige 
Teil des Cuprokupfers, welches als komplexes Salz vorhanden, in Abzug gebracht wird.

Da Cuprochlorid sich in Lösungen von Salzsäure und Chloriden löst, ist es als sicher anzusehen, dafs die Chlorionen das Cuprochlorid als neutralen Teil addieren und damit komplexe Anionen bilden. Es mufs sich ein bestimmtes Gleichgewicht zwischen den freien Chlorionen und den kupferhaltigen Anionen bei Gegenwart von festem Cuprochlorid herstellen. Sind die Bedingungen dieses Gleichgewichtes, insbesondere auch seine Konstanten bekannt, so läIst sich daraus berechnen, wie viel von der komplexen Cuproverbindung neben einer bestimmten Menge freier Chlorionen sich in der mit Cuprochlorid gesättigten Lösung befindet, wobei es gleichgültig ist, ob die Chlorionen von der Dissoziation von Chlorkalium oder von Salzsäure oder wie in unserem Falle von der Dissoziation des Cuprichlorids oder des freien Cuprochlorids herrühren.

Quantitative Bestimmungen der Löslichkeit von Cnprochlorid in Salzsäure rühren, wie oben erwähnt, von Le Chatelder und ENGEL her. Beide Forscher untersuchten die Löslichkeit in recht konzentrierten Salzsäurelösungen; die geringste angewandte Salzsäurekonzentration betrug 0.8975 normal, die höchste 10.4 normal. Wenn wir nur die kleineren Konzentrationen in Betracht ziehen, für welche eine Veränderung des Lösungsmittels noch nicht angenommen zu werden braucht, so lassen sich die Resultate in der folgenden Tabelle zusammenstellen.

Tabelle 2.

Löslichkeit von Cuprochlorid in Salzsäure.

\begin{tabular}{|c|c|c|c|c|}
\hline Autor & $\begin{array}{c}\text { Salzsäure- } \\
\text { konzentration a }\end{array}$ & $\begin{array}{l}\text { Cuprochlorid- } \\
\text { konzentration b }\end{array}$ & $\frac{\mathrm{a}}{\mathrm{b}}$ & $\frac{\mathrm{a}^{2}}{\mathrm{~b}}$ \\
\hline Le Chatelier & 0.8975 & 0.0475 & 18.9 & 17 \\
\hline Le Chatelier & 1.57 & 0.14 & 11.2 & 17.6 \\
\hline EngeL . . & 1.75 & 0.15 & 11.7 & 20.4 \\
\hline Le Chateuter & 1.82 & 0.1575 & 11.6 & 21.1 \\
\hline Enael . . & 2.86 & 0.29 & 8.97 & 23.3 \\
\hline Le Chatelier & 3.45 & 0.45 & 7.75 & 26.4 \\
\hline
\end{tabular}

Die Konzentrationen sind in Grammmolekülen im Liter angegeben, wobei für Cuprochlorid die Formel $\mathrm{CuCl}$ angenommen wurde. Wie die vorletzte Spalte der Tabelle ergiebt, erfolgt die 
Zunahme des gelösten Cuprochlorids nicht dem Chlorgehalt proportional, sondern schneller als dieser; es besteht aber auch keine Proportionalität des gelösten Kupfers mit dem Quadrate der Chlorkonzentrationen. Es ist also in keinem Fall zulässig, aus diesen Bestimmungen auf die Menge des Cuprochlorids zu extrapolieren, die in Form komplexer Ionen in den an Chlor weit ärmeren Lösungen vorhanden sind.

Besser für unsere Berechnung eignen sich die Bestimmungen, welche ABEL kürzlich veröffentlicht bat. Auch ABEL bestimmte die Löslichkeit des Cuprochlorids in Salzsäure, ging aber dabei von kleineren Konzentrationen aus. Die niedrigste Konzentration war 0.04222 , die böchste 2.7 normal. Die Bestimmungen beziehen sich auf $15^{\circ} \mathrm{C}$. Die Sättigung der Lösung mit Cuprochlorid suchte ABEn dadurch zu erreichen, dals er das Cuprochlorid erst mit konzentrierter Salzsäure schüttelte und die klare Lösung mit Wasser: versetzte. Es scheidet sich hierbei Cuprochlorid aus; er liefs absetzen und pipettierte einen Teil der lösung für die Analyse ab. Er glaubte dabei wirklich das Gleichgewicht erhalten zu haben, weil dieses von oben, d. h. durch Ausscheidung von Salz aus einer übersättigten Lösung sich einstellte. Da er aber nach dem Versetzen mit Wasser nicht mehr schüttelte, sondern nur absetzen liefs, ist zwar Untersättigung ausgeschlossen, wohI aber eine Übersättigung möglich. Bedenklicher ist ferner, dafs zwar mit ausgekochtem Wasser und Salzsäure gearbeitet wurde, aber bei der Entnahme der Proben und der Titrierung die Oxydation durch den Luftsauerstoff nicht genügend ausgeschlossen war. Die Bestimmung des gelösten Cuprochlorids erfolgte durch Titration mit Kaliumpermanganat; es sind hierbei zwei Fehler möglich, einmal ein Verbrauch von Permanganat durch Oxydation der Salzsäure unter Entbindung von Chlor und ferner dadurch, dafs lange Zeit anhaltende Rötung durch Permanganat eintritt, noch ehe alles Cuprochlorid oxydiert ist. Wartet man das Verschwinden der Rötung ab, so bleibt unsicher, ob die Entfärbung durch die Einwirkung der Salzsäure oder durch die des Cuprochlorids bewirkt wird.

Da diese Fehlerquellen gerade bei kupferarmen Lösungen einen erheblichen Einflufs iiben, erklärt es sich, dafs für das Gleichgewicht zwischen Salzsäure und Cuprochlorid ABEL gerade bei den verdünntesten Lösungen -0.04222 bis 0.63 normal $\mathrm{HCl}$ — „Kon-

1 Vergl. Caven und HiLl, Journ. Soc. Chem. Ind. 16 (1897), 981-983. 
stanten" erhielt, die von den übrigen sehr erbeblich abweichen und auch mit unseren Resultaten am wenigsten im Einklang stehen.

ABEL prüfte, ob ein konstantes Verhältnis zwischen dem gelösten komplexen Salz und der ersten oder zweiten Potenz der freien Salzsäure besteht. Er findet; dals nur bei höheren Konzentrationen der Salzsäure zwischen $0.5-2$ normal eine angenäherte Konstanz für den Ausdruck $\mathrm{Cl}: \sqrt{\mathrm{CuCl}}$ besteht, bei niedrigeren Konzentrationen steigt dieser Ausdruck mit der Konzentration an, während der Ausdruck $\mathrm{Cl}$ : $\mathrm{CuCl}$ langsam abnimmt.

Bei der theoretischen Untersuchung dieses Gleichgewichtes zwischen Cuprochlorid und Salzsäure kommt ABEL zu dem Schlufs, dals die Eormel der freien Cuproionen aus diesen Versuchen nicht ermittelt werden kann, dal's es also unentschieden ist, $a b$ ihnen die Formel $\mathrm{Cu}^{\cdot}$ oder $\mathrm{Cu}_{2}{ }^{\prime}$ zuzuschreiben ist. Diese Folgerung ist richtig. Nicht richtig ist es dagegen, dals das Gleichgewicht über die Zusammensetzung der komplexen Verbindung vollkommenen Aufschluis erteilen kann. Die komplexen Verbindungen aus Cuprochlorid und Salzsäure entstehen, indem sich ein oder mehrere Moleküle Salzsäure an ein oder mehrere Moleküle Cuprochlorid anlagern. Die Anionen entstehen durch Anlagerung von ein oder mehreren Chlorionen an ein oder mehrere Moleküle Cuprochlorid. Wir können die Bildung der komplexen Anionen ausdrücken durch die Gleichung:

$$
\begin{aligned}
m \mathrm{CuCl}+n \mathrm{Cl}^{\prime} & =(\mathrm{CuCl})_{m} \cdot \mathrm{Cl}_{n} \text { oder } \\
(\mathrm{CuCl})_{m}+n \mathrm{Cl}^{\prime} & =(\mathrm{CuCl})_{m} \cdot \mathrm{Cl}_{n}
\end{aligned}
$$

wo $m$ und $n$ ganze Zahlen sein müssen. Die Gleichgewichtsbedingung ist im ersteren Falle:

$$
[\mathrm{CuCl}]^{m} \cdot[\mathrm{Cl}]^{n}=k \cdot\left[(\mathrm{CuCl})_{m} \cdot \mathrm{Cl}_{n}\right]
$$

im zweiten Falle:

$$
\left[(\mathrm{CuCl})_{m}\right] \cdot(\mathrm{Cl})^{n}=k\left[(\mathrm{CuCl})_{m} \cdot \mathrm{Cl}_{n}\right]
$$

Da bei den Löslichkeitsversuchen Cuprochlorid Bodenkörper ist, geht seine aktive Masse in die Konstante ein und wir erbalten aus beiden Gleichungen dieselbe Beziehung:

$$
\frac{[\mathrm{Cl}]^{n}}{\left[(\mathrm{CuCl})_{m} \cdot \mathrm{Cl}_{n}\right]}=k
$$


Experimentell kann man nur ermitteln, welchen Wert $n$ besitzt, d. h. ob die erste, zweite oder $n$ te Potenz der Chlorkonzentrationen dem in Form der komplexen Anionen gelösten Kupfer proportional ist. Die Formel des Cuprochlorids und damit die Formel der freien Cuproionen ist auf diesem Wege, wie auch ABEL auf andere Weise nachwies, nicht zu ermitteln. Aber auch die Formel des komplexen Salzes wird auf diese Weise nicht vollständig festgestellt, da die Konstanz der Beziehung gänzlich unentschieden lälst, welchen Wert $m$ besitzt. Nur über die Anzahl $n$ der von einem Molekül Kupferchlorür addierten Chloratome in dem komplexen Anion giebt die Konstanz irgend eines Ausdruckes Aufschlufs. Wenn ABEL aus der Konstanz des Ausdruckes: $\mathrm{Cl}^{2}$ : $\mathrm{Cu}$ bei höheren Salzsäurekonzentrationen schliefst, dafs dem komplexen Anion die Formel $\mathrm{Cu}_{2} \mathrm{Cl}_{4}{ }^{\prime \prime}$ zukommen müsse, so ist das irrig; es kann nur geschlossen werden, dafs ihnen die Formel: $(\mathrm{CuCl})_{m} \mathrm{Cl}_{2}$ zukommt, wobei $m$ jede beliebige ganze Zahl, die Formel also $\mathrm{CuCl}_{3}{ }^{\prime}$ oder $\mathrm{Cu}_{2} \mathrm{Cl}_{4}{ }^{\prime \prime}$ oder $\mathrm{Cu}_{3} \mathrm{Cl}_{5}{ }^{\prime \prime}$ sein kann. In Wirklichkeit trifft nicht die von ABEL angenommene Formel, sondern die Formel $\mathrm{CuCl}_{3}{ }^{\prime} \mathrm{zu}$, wie weiter unten gezeigt wird.

Bei geringen Salzsäurekonzentrationen ist der Ausdruck $\mathrm{Cl}: \mathrm{Cu}$ konstant, daraus folgt nicht notwendig, dal's bei kleinen Salzsäurekonzentrationen die Formel der komplexen Anionen $\mathrm{CuCl}_{2}{ }^{\prime}$ sein muls: sie kann auch $\mathrm{Cu}_{2} \mathrm{Cl}_{3}{ }^{\prime}$ oder $\mathrm{Cu}_{3} \mathrm{Cl}_{4}{ }^{\prime}$ u. s. w. sein.

Um die Menge des bei verschiedenen Konzentrationen der Chlorionen gelösten Cuprochlorids zu bestimmen, wurden in der oben angegebenen Weise luftfreie Chlorkaliumlösungen bekannter Konzentrationen mit Cuprochlorid 6-8 Stunden lang in einer Kohlensäureatmosphäre geschüttelt. Da dem Cuprochlorid von der vorhergehenden Behandlung mit Wasser immer eine gewisse Menge metallischen Kupfers beigemengt war, konnte eine Bildung von Cuprichlorid durch Oxydation nicht stattfinden, selbst wenn Spuren von Sauerstoff Zutritt gefunden hätten. Auch durch Zersetzung von Cuprochlorid entsteht kein Cuprichlorid, wenn die Chlorkaliumkonzentration 0.1 normal und höher ist. Das ergab die Prüfung mit Jodkalium, wodurch keine Spur von Jod aus den Lösungen entbunden wird. Wenn keine mefsbaren Mengen von Cupriionen vorhanden sind, können sich auch keine mefsuaren Mengen von freien Cuproionen in der Lösung befinden. Das gesamte Kupfer mufs deshalb in der Lösung in Form des komplexen Salzes und seiner Ionen zugegen sein. 
Tabelle 3.

Löslichkeit von Cuprochlorid in konzentrierteren Chlorkaliumlösungen.

\begin{tabular}{l|c|c|c|c|c|c}
\hline \hline Nr. & $\begin{array}{c}\text { Tempe- } \\
\text { ratur }\end{array}$ & $\begin{array}{c}\text { Chlorkalium } \\
\text { g-Mol }\end{array}$ & $\begin{array}{c}\text { Kupfer } \\
\text { g-Atom }\end{array}$ & $\mathrm{K}_{1}$ & $\mathrm{~K}_{2}$ & $\mathrm{~K}_{8}$ \\
\cline { 3 - 6 } & & \multicolumn{2}{|c|}{ im Liter } & & \\
\hline \hline 1 & 18.3 & 0.05 & 0.002411 & 19.74 & 0.940 & 0.848 \\
2 & 16 & 0.1 & 0.004702 & 20.27 & 1.931 & 1.75 \\
3 & 16 & 0.2 & 0.009458 & 20.15 & 3.84 & 3.47 \\
4 & 19.2 & 1.0 & 0.0970 & 9.31 & 8.40 & 6.70 \\
5 & 16.4 & 2.0 & 0.3840 & 4.21 & 6.80 & 3.95
\end{tabular}

Es war zu prüfen, ob der Ausdruck $\frac{\left[\mathrm{Cl}^{\prime}\right]}{[\overline{\mathrm{Cu}}]}$ oder $\frac{\left[\mathrm{Cl}^{\prime}\right]^{2}}{\left[\mathrm{Cu}^{2}\right.}$ oder ein anderer konstant ist.

Wenn das Cuprochlorid nur ein Chlorion addiert, also ein einwertiges Anion $(\mathrm{CuCl})_{m} \mathrm{Cl}^{\prime}$ bildet, so ist in der gemeinsamen Lösung das $\mathrm{Salz} K(\mathrm{CuCl})_{m} \mathrm{Cl}$ ebenso stark dissoziiert wie das Chlorkalium. Wir können also für das Verhältnis der Anionen das Verhältnis der Gesamtkonzentrationen der Salze setzen, wobei von dem angewandten Chlorkalium der Teil abzuziehen wäre, der in Form des komplexen Salzes in der Lösung vorhanden ist. $\mathrm{Da} m$, wie aus unten zu beschreibenden elektrometrischen Messungen sich ergiebt, gleich 1 ist, so müssen wir für jedes gelöste Kupferatom ein Chlorkaliumatom abziehen, es ist also die Konstante:

$$
\frac{\left[\mathrm{Cl}^{\prime}\right]}{\left[(\mathrm{CuCl})_{m} \mathrm{Cl}^{\prime}\right]}=\frac{[\mathrm{KCl}]-[\mathrm{Cu}]}{[\mathrm{Cu}]}=\mathrm{K}_{1}
$$

Die Werte von $K_{1}$ sind in der fünften Spalte der Tabelle angegeben. Sie sind bis zu einer Chlorkaliumkonzentration von 0.2 konstant. Dals $\mathrm{K}_{1}$ in der 0.05 normalen Lösung etwas kleiner ist, liegt daran, dals in ihr nicht das gesamte Kupfer als komplexes Salz vorhanden ist. Vielmehr läfst sich in dieser Lösung mit Jodkalium noch die Gegenwart von Cuprichlorid qualitativ und quantitativ nachweisen und ihr entspricht eine kleine Menge freier Cuproionen. Es ist also der Nenner etwas zu grofs, da in ihm die Gesamtkupfermenge eingesetzt ist, wodurch es sich erklärt, dafs der Quotient etwas zu klein ist.

Z. anorg. Chem. XXXI. 
Jedenfalls ist zu schliefsen, dafs bis zu Konzentrationen von 0.2 Chlorkalium ein Chlorion mit dem Cuprochlorid verbunden ist, wobei es unentschieden bleibt, ob letzteres die Formel $\mathrm{CaCl}, \mathrm{Ca}_{2} \mathrm{Cl}_{2}$, $\mathrm{Cu}_{3} \mathrm{Cl}_{3}$ u. s. w. besitzt, ob also dem komplexen Anion die Formel $\mathrm{CuCl}_{2}{ }^{\prime}$ oder $\mathrm{Cu}_{2} \mathrm{Cl}_{3}{ }^{\prime}$ oder $\mathrm{Cu}_{3} \mathrm{Cl}_{4}{ }^{\prime}$ zukommt.

In den normalen und doppelt normalen Chlorkaliumlösungen muls dagegen ein Anion von anderem Typus vorhanden sein. Die einfachste Annahme ist, dals diesem die Formel:

$$
\mathrm{CuCl}_{3} \text { " oder } \mathrm{Cu}_{2} \mathrm{Cl}_{4}^{\prime \prime}
$$

zukommt, dafs mit einem Molekül des einfachen oder polymerisierten Cuprochlorids zwei Chlorionen verbunden sind. Es mufs dann nach dem Massenwirkungsgesetz die Beziehung gelten:

$$
\frac{(\mathrm{Cl})^{2}}{\left[\mathrm{CuCl}_{3}\right]}=\mathrm{K}_{2}
$$

wobei $\mathrm{Cl}$ die freien Chlorionen, $\mathrm{CuCl}_{3}$ die freien komplexen Ionen bedeuten. Diese Gleichung wird auch nicht verändert, wenn den komplexen Anionen die Formel $\mathrm{Cu}_{2} \mathrm{Cl}_{4}{ }^{\prime \prime}$ zukäme. Wenn wir aber von den Ionen zu dem Gesamtsalz übergehen, so ergeben die beiden Annahmen verschiedene Werte. Ist $\alpha_{1}$ der Dissoziationsgrad des Chlorkaliums, so können wir [KCl.cc], für [Cl] einsetzen, wobei $[\mathrm{KCl}]$ das gesamte nicht zur Bildung des komplexen Salzes verwendete Chlorkalium bedeutet. Für $\left[\mathrm{CuCl}_{3}\right]$ können wir $\left[\mathrm{Cu} . \alpha_{2}\right]$ einsetzen, wo $[\mathrm{Cu}]$ das gesamte Kupfer, $\alpha_{2}$ den Dissociationsgrad des komplexen Salzes bedeutet. Wir erhalten also:

$$
\frac{[\mathrm{KCl}]^{2} \cdot\left[\alpha_{1}\right]^{2}}{[\mathrm{Cu}] \cdot\left[\alpha_{2}\right]}=k_{2}
$$

In jedem Falle ist das komplexe Salz zwei- und einionig, sei es, dafs es die Formel: $\mathrm{K}_{2} \mathrm{CuCl}_{3}$, sei es, dafs es die Formel: $\mathrm{K}_{2} \mathrm{Cu}_{2} \mathrm{Cl}_{4}$ besitzt.

Es ergiebt sich nun beim Vergleich der Dissoziationsgrade der Salze rom Typus des Chlorkaliums mit denen eines zwei- und einionigen Salzes, dafs bei gleicher Konzentration das Quadrat des einen der ersten Potenz des anderen gleich ist. Das folgt z. B. aus der nachstehenden, nach den Zahlen von КонJ.Radsch berechneten Tabelle, in welcher als Dissoziationsgrade von $\mathrm{RCl}_{2}$ die Mittelwerte der Dissoziationsgrade von Chlorbarium und Chlorzink aufgeführt sind. 
Tabelle 4 .

\begin{tabular}{|c|c|c|c|}
\hline \multirow{2}{*}{ Konzentration } & \multicolumn{2}{|c|}{$\mathrm{KCl}$} & \multirow{2}{*}{$\mathrm{RCl}_{2}$} \\
\hline & $\alpha_{1}$ & $\alpha_{1}^{2}$ & \\
\hline 0.01 & 0.938 & 0.880 & 0.87 \\
\hline 0.05 & 0.880 & 0.774 & 0.768 \\
\hline 0.1 & 0.855 & 0.731 & 0.737 \\
\hline 0.5 & 0.780 & 0.608 & 0.602 \\
\hline
\end{tabular}

Machen wir nun die Annahme, dals in einer Lösung, die einund einionige neben ein- und zweiionigen Salzen enthält, der Dissoziationsgrad jedes von beiden so ist, wie wenn die Gesamtkonzentration nur von diesem Salz herrührte, so mürste in unserem Falle der Dissoziationsgrad $\alpha_{2}$ des komplexen Kupfersalzes dem Quadrat des Dissoziationsgrades $\alpha_{1}$ des Chlorkaliums gleich sein; wir erhielten also:

$$
\frac{[\mathrm{KCl}]}{[\mathrm{Cu}]}=\mathrm{K}_{2} \text {. }
$$

Die Menge des nicht zur Bildung des Doppelsalzes verbrauchten Chlorkaliums ist gleich dem angewandten Chlorkalium rermindert um die einfache oder doppelte Konzentration des gelösten Kupfers, je nachdem dieses in Form des Salzes $\mathrm{K}_{2} \mathrm{CuCl}_{3}$ oder $\mathrm{K}_{2} \mathrm{Cu}_{2} \mathrm{Cl}_{4}$ vorhanden ist. Im ersten Falle müfste also konstant sein: $\frac{[\mathrm{KCl}-\mathrm{Cu}]^{2}}{[\mathrm{Cu}]}$ im anderen Falle $\frac{[\mathrm{KCl}-2 \mathrm{Cu}]^{2}}{[\mathrm{Cu}]}$, wo $\mathrm{KCl}$ diesmal die gesamte Menge des angewandten Chlorkaliums bedeutet.

In der Tabelle 3 sind unter $K_{2}$ und $K_{3}$ die nach beiden Annahmen berechneten Werte der Konstanten eingetragen. Wir erkennen, dafs bei kleineren Konzentrationen die Werte von $\mathrm{K}_{2}$ und $\mathrm{K}_{3}$ stark ansteigen, dafs also in diesen Lösungen keinesfalls zwei Moleküle Chlorkalium sich mit einem Molekül Cuprochlorid vereinigen. Aber auch bei den höheren Konzentrationen ergiebt sich weder für die eine noch für die andere Annahme eine Konstanz des theoretischen Ausdrucks.

Immerhin zeigen die Werte von $\mathrm{K}_{2}$ für normale und doppeltnormale Lösung eine gröfsere Annäherung aneinander, als die von $K_{1}$ und $K_{3}$. Dals die Konstanz nicht vollständig ist, liegt daran, dafs unsere Annahme über die Dissoziationsgrade der Salze in der gemischten Lösung ungenau ist. 
Die elektrometrischen Messungen, über die weiter unten berichtet wird, ergeben mit grölster Sicherheit, dafs in konzentrierten Lösungen die Formel des komplexen Salzes $\mathrm{K}_{2} \mathrm{CuCl}_{3}$ ist.

Nachdem ermittelt worden ist, wie viel Cuprochlorid bei einem bestimmten Gehalt an Chlorionen unter Bildung komplexer Anionen addiert wird, können wir berechnen, wie viel von dem Cuprochlorid der reinwässerigen Lösung in Form von undissoziierten Salz, wie viel in Form von freien Cuproionen und wie viel in Form des komplexen Salzes bezw. seiner Anionen vorhanden ist. Der Gesamtchlorgehalt der reinwässerigen Lösung ist 0.005436 normal, die komplexen Anionen haben demnach in diesen Lösungen sicher die Formel $(\mathrm{CuCl})_{m} \mathrm{Cl}^{\prime}$, da sich erst bei Chlorkonzentrationen von mehr als 0.5 die Gegenwart der höheren Komplexe $\mathrm{CuCl}_{3}{ }^{\prime \prime}$ bemerkbar macht. Wie wir oben gesehen haben, ist das Verbältnis $\mathrm{Cl}^{\prime}:(\mathrm{CuCl})_{m} \mathrm{Cl}^{\prime}$ für 0,1 normale Chlorkaliumlösungen 20.27, für 0.2 normale 20.15, im Mittel also 20.2. Die Konzentration der Chlorionen in der reinwässerigen Lösung ergiebt sich aus der Gesamtkonzentration des Chlors und dem Dissoziationsgrad der einzelnen Salze. Es sind von den 0.005436 Grammatomen Chlor(S.12) 0.004444 in Form von Cuprichlorid vorhanden, 0.000363 in Form von Salzsäure und der Rest 0.000629 in Form von komplexen und freien Cuproverbindungen. Enthielte die Lösung nur Cuprichlorid, so wäre der Dissoziationsgrad nach der Analogie mit $\mathrm{ZnCl}_{2}$ und $\mathrm{BaCl}_{2}$ etwa 0.9. Unsicher ist es, ob dabei das Cuprichlorid nur in $\mathrm{Cu}^{2}$ und $2 \mathrm{Cl}^{\prime}$ zerfällt oder auch zum Teil in $\mathrm{CuCl}^{\prime}$ und $\mathrm{Cl}^{\prime}$ oder in $2 \mathrm{CuCl}_{3}{ }^{\prime}$ und $\mathrm{Cu}^{*}$. Wenn nur die Dissoziation nach dem ersten Schema verläuft, würden vom Cuprichlorid geliefert werden $2 \cdot 0.002222 \cdot 0.9=0.004$ Chlorionen. Die Salzsäure kann als vollständig dissoziiert angesehen werden, sie liefert 0.000363 Chlorionen. Das freie Cuprochlorid würde, nach der Analogie mit dem Chlorkalium zu schliefsen, in einer Lösung, die 0.005436 Chlor enthält, zu 0.955 dissoziiert sein. Setzen wir die Menge des in Form der komplexen Anionen vorhandenen Kupfers $=x$, so ist die Menge des freien Cuprochlorids 0.000629-x und die Menge der von ihnen gelieferten Chlorionen $(0.000629-x)$. 0.955. Die Gesamtmenge der freien Chlorionen ist also:

$0.004+0.000363+(0.000629-x) \cdot 0.955,=0.00498-0.955 x$.

Die Menge der komplexen Ionen ist $x$, also ist:

$$
\frac{0.00498-0.955 x}{x}=20.2 \quad x=0.00023 \text {. }
$$


Da anzunehmen ist, dafs das Salz ebenso stark dissoziiert ist, wie Chlorkalium in einer Lösung, die 0.005436 Chlor enthält, also zu 0.955, so ist die Menge des gesamten komplexen Salzes $=0.000241$. Die Menge des gesamten freien Cuprochlorids ist also: 0.000629 $0.000241=0.000388$. Die Menge der gesamten freien Chlorionen ist $0.00498-0.955 \cdot 0.00023=0.00476$. Die Menge der freien Cuproionen ist $=0.000388 \cdot 0.955=0.000371$, wenn das freie Cuprochlorid die Formel $\mathrm{CuCl}$ besitzt und ebenso dissoziiert ist wie $\mathrm{KCl}$ in entsprechender Lösung. Wenn dagegen das freie Cuprochlorid die Formel $\mathrm{Cu}_{2} \mathrm{Cl}_{2}$ besitzt, ist es ebenso wie das Cuprichlorid in dieser Lösung zu 0.90 dissoziiert; die Menge der Cuproionen $\mathrm{Cu}_{2}$ " wäre dann: $1 / 2 \cdot 0.000388 \cdot 0.9=0.0001746$.

Es wurde versucht, die Frage, ob die freien Cuproionen die Formel $\mathrm{Cu}$ oder $\mathrm{Cu}_{2}{ }^{*}$ besitzen, experimentell durch Untersuchung der Löslichkeit von Cuprochlorid bei Gegenwart eines Überschusses von Cupri- oder Chlorionen zu entscheiden.

\section{Löslichkeit von Cuprochlorid in Gegenwart überschüssiger Cupriionen.}

Es ist zwischen den beiden Annahmen zu entscheiden, ob die freien Cuproionen die Formel $\mathrm{Cu}^{*}$ oder $\mathrm{Cu}_{2}{ }^{*}$ besitzen.

Für die Spaltung der Cuprosalze in Kupfer und Cuprisalze ergiebt sich dann entweder die Gleichung:

$$
2 \mathrm{Cu}^{*}=\mathrm{Cu}^{*}+\mathrm{Cu} \text { oder } \mathrm{Cu}_{2}=\mathrm{Cu}^{*}+\mathrm{Cu} \text {. }
$$

daraus folgt nach dem Massenwirkungsgesetz entweder:

$$
\frac{\left(\mathrm{Cu}^{\circ}\right)^{2}}{\mathrm{Cu}{ }^{*}}=k \text { oder } \frac{\left(\mathrm{Cu}_{2}{ }_{2}\right)}{\mathrm{Cu}^{*}}=k \text {. }
$$

Wenn also bei Gegenwart von metallischem Kupfer und wechselnden Mengen von Cupriionen ein Cuprosalz in Lösung gebracht wird, - wobei die Lösung für das Cuprosalz nicht gesättigt zu sein braucht, - so müfste sich aus der Konstanz für den einen oder anderen Ausdruck eine Entscheidung unserer Frage ergeben.

Es wurde deshalb die Löslichkeit des Cuprochlorids bei Gegenwart von metailischem Kupfer bestimmt, wobei die Menge der Cupriionen durch Zusatz wechselnder Mengen Cuprisulfat variiert 
wurde. Es wurde wie in den oben angeführten Versuchen die Menge des Cuprikupfers, des Gesamtkupfers und des Chlors quantitativ bestimmt, die Menge des Cuprokupfers aus der Differenz des Cuprikupfers und des Gesamtkupfers ermittelt. Die Resultate sind in der folgenden Tabelle zusammengestellt:

\section{Tabelle, 5 .}

Löslichkeit von Kupferchlorür in Kupfersulfatlösungen.

Milligrammmoleküle im Liter.

\begin{tabular}{c|c|c|c|c|c|c}
\hline \hline Nr. & $\begin{array}{c}\text { Tempe- } \\
\text { ratur }\end{array}$ & $\begin{array}{c}\text { Konz. des } \\
\text { Cuprisulfats }\end{array}$ & $\begin{array}{c}\text { Cupri- } \\
\text { kupfer }\end{array}$ & $\begin{array}{c}\text { Gesamt- } \\
\text { kupfer }\end{array}$ & $\begin{array}{c}\text { Cuprokupfer } \\
\text { berechnet }\end{array}$ & $\begin{array}{c}\text { Chlor } \\
\text { gefunden }\end{array}$ \\
\hline \hline & 19.7 & - & 2.258 & 2.880 & 0.622 & 5.312 \\
2 & 16.3 & 0.49375 & 2.746 & 3.125 & 0.379 & 4.805 \\
3 & 18.6 & 0.9875 & 3.145 & 3.602 & 0.457 & 4.908 \\
4 & 17.5 & 1.4812 & 3.315 & 3.915 & 0.600 & 4.530 \\
5 & 19.4 & 1.975 & 4.131 & 4.553 & 0.422 & 4.687 \\
6 & 20.4 & 2.4687 & 4.349 & 4.786 & 0.437 & 4.287 \\
7 & 20.5 & 2.9625 & 4.625 & 5.193 & 0.509 & 4.256 \\
8 & 20.1 & 4.9375 & 6.546 & 7.276 & 0.730 & 4.329
\end{tabular}

Die Menge des gelösten Cuprochlorids steigt nicht beständig bei zunehmenden Cuprigehalt der Lösung, sondern sie fällt und steigt in scheinbar unregelmäfsiger Weise. Das liegt zum Teil daran, dals bei Zunahme der Cupriionen zwar die Menge der Cuproionen steigen muls, dals aber andererseits dadurch, dals Cupriionen durch das Cuprisulfat geliefert werden, die Menge des Cuprichlorids abnehmen muls; damit fällt die Menge der Chlorionen und hierdurch die Menge der komplexen Ionen $\mathrm{CuCl}_{2}{ }^{\prime}$, so dafs das Steigen der freien Cuproionen einer Abnahme der komplexen Ionen entspricht. Der Gesamtgehalt an Cuprokupfer kann also fallen, weil die Menge der komplexen Ionen abnimmt, obwohl die Menge der Cuproionen zunimmt. Da auch noch andere Störungen z. B. in Folge der Bildung komplexer Cuprosulfationen möglich sind, so erscheint eine Berechnung der Menge der freien Cuproionen und damit eine Prüfung der theoretischen Formel unmöglich.

Unabhängig von jeder Annahme über die Formel der freien Cuproionen mü̈ste sich noch eine Beziehung zwischen den Meligen der Cupriionen und der Chlorionen ergeben. 
Sei die Formel des undissoziierten Cuprochlorids $(\mathrm{CuCl})_{n}$, so kann dessen Zerfall in Cuprichlorid und Kupfer durch die Gleichung ausgedrückt werden.

$$
2(\mathrm{CuCl})_{n}=n \mathrm{CuCl}_{2}+n \mathrm{Cu} .
$$

Wenn festes Cuprochlorid und metallisches Kupfer Bodenkörper sind, muls die Menge des undissoziierten Cuprichlorids konstant sein und damit das Produkt $\left(\mathrm{Cu}^{*}\right)\left(\mathrm{Cl}^{\prime}\right)^{2}{ }^{1}$ Die Tabelle ergiebt in der That, dafs der Chlorgehalt fällt, während der Cuprigehalt zunimmt. Wäre alles Cuprikupfer und alles Chlor in Form freier Ionen in der Lösung vorhanden, so mülste, wenn der Gehalt an Cuprikupfer von 2.258 auf 4.625 also etwa auf das doppelte steigt (Versuch 1 und 7), der Chlorgehalt von 5.3 auf 2.6 fallen; er fällt aber nur auf 4.25 .

Eine quantitative Bestätigung ergiebt sich auch nicht angenähert, selbst wenn das in Form der komplexen Ionen $\mathrm{CuCl}_{2}^{\prime}$ gebundene Chlor berücksichtigt wird.

Der Grund liegt darin, dals die Dissoziation des Cuprichlorids und noch mehr die des Cuprisulfats ziemlich gering ist und sich bei steigender Konzentration sehr ändert. Eine genaue Berechnung des Dissoziationsgrades, die schon bei Salzen vom Typus des Chlorkaliums sehr schwer ist, ist bei Salzen wie das Cuprichlorid und Cuprisulfat noch dazu in gemischten Lösungen zur Zeit unmöglich.

\section{Löslichkeit des Cuprochlorids bei Zusatz von Chlorkalium.}

Etwas einfacher liegen die Verhältnisse, wenn nur ein- und zweiwertige neben ein- und einwertigen Salzen vorhanden sind. Bestimmen wir die Löslichkeit des Cuprochlorids in Gegenwart von Chlorkalium, so wird durch die Vermehrung der Chlorionen die Menge der Cuproionen verringert werden. Da Cuprochlorid Bodenkörper ist, so mufs entweder das Produkt $\left(\mathrm{Cu}^{*}\right)\left(\mathrm{Cl}^{\prime}\right)$ oder $\left(\mathrm{Cu}_{2}{ }_{2}\right)\left(\mathrm{Cl}^{\prime}\right)^{2}$ konstant sein, je nachdem die freien Cuproionen ein- oder zweiatomig sind. Das Experiment lälst also hier die Entscheidung zu. Auch auf einem anderen Wege mulsten dieselben Versuche eine Entscheidung bringen. Es ist in jedem Fall neben dem Cuprochlorid metallisches Kupfer Bodenkörper. Das Verhältnis zwischen Cupro- und Cupriionen mufs ebenso, wie oben dargelegt wurde, eine Entscheidung geben, ob die

\footnotetext{
1 Auf anderen Wege ist ABEL zu demselben Schlusse gelangt.
} 
Cuproionen eill- oder zweiatomig sind. Ferner muls unabhängig hiervon in jedem Falle das Produkt aus der Konzentration der Cupriionen in das Quadrat der Chlorionen konstant sein. Die nachfolgende Tabelle giebt die experimentell gefundenen Zahlen.

\section{Tabelle 6 .}

Jöslichkeit von Kupferchlorür in Gegenwart wechselnder Mengen Chlorkalinm.

Konzentrationen in Millimolen.

\begin{tabular}{|c|c|c|c|c|c|c|}
\hline $\mathrm{N} r$. & $\begin{array}{c}\text { Tempe- } \\
\text { ratur }\end{array}$ & $\mathrm{KCl}$ & Cuprikupfer & $\begin{array}{l}\text { Gesamt- } \\
\text { kupfer }\end{array}$ & $\begin{array}{c}\text { Cuprokupfer } \\
\text { berechnet }\end{array}$ & Chlor \\
\hline 1 & $20^{\circ}$ & 0 & 2.222 & 2.851 & 0.629 & 5.436 \\
\hline 2 & 19 & 1 & 1.901 & 2.385 & 0.484 & 5.287 \\
\hline 3 & 19 & 2 & 1.571 & 2.150 & 0.589 & 5.614 \\
\hline 4 & 19 & 2.5 & 1.421 & 1.955 & 0.534 & 6.015 \\
\hline 5 & 19 & 3 & 1.523 & 1.983 & 0.460 & 6.247 \\
\hline 6 & 16 & 5 & 1.008 & 1.522 & 0.514 & 7.525 \\
\hline 7 & 18 & 10 & 0.475 & 1.236 & 0.761 & 11.785 \\
\hline 8 & 20 & 15 & 0.322 & 1.344 & 1.022 & 16.437 \\
\hline 9 & 19 & 20 & 0.324 & 1.446 & 1.122 & 21.356 \\
\hline 10 & 19 & 30 & 0.1308 & 1.761 & 1.630 & 31.911 \\
\hline 11 & 18 & 50 & 0.1088 & 2.411 & 2.302 & \\
\hline 12 & 16 & 100 & 0 & 4.702 & 4.702 & $\leftrightarrow \stackrel{艹}{\exists}$ \\
\hline 13 & 16 & 200 & 0 & 9.485 & 9.485 & 록 \\
\hline 14 & 19 & 1000 & 0 & 97.0 & 97.0 & $=8$ \\
\hline 15 & 16 & 2000 & 0 & 384.0 & 384.0 & \\
\hline
\end{tabular}

Eine Schwierigkeit bietet auch hier die Berechnung der freien Cupri- und Cuproionen und auch die der Chlorionen. Wenn alle in den Lösungen vorhandenen Chloride gleich stark dissoziiert wären wie Chlorkalium, so könnte man aus dem Gesamtchlorgehalt der Lösung die Menge der komplexen Cuproverbindung berechnen. Es müfste nach der oben S. 20 gefundenen Beziehung $(\mathrm{Cl})-2\left(\mathrm{KCuCl}_{2}\right)$ $=20.2$ sein, woraus sich ergiebt $(\mathrm{Cl}):\left(\mathrm{KCuCl}_{2}\right)=22.2$. Nach dieser Formel wurde aus dem Gesamtchlorgehalt die Gesamtmenge des komplexen Cuprosalzes berechnet. Diese Berechnung istungenau, weil das in den Lösungen immer vorhandene Cuprichlorid schwächer dissoziiert ist als das Chlorkalium und sein Chlor deshalb voranssichtlich weniger komplexes Salz bildet. Eine genauere Berechnungsweise konnte aber nicht angewandt werden, weil der Dissnziationsgrad des Cuprichlorids 
insbesondere bei Gegenwart von Chlorkalium nicht bekannt ist. Aus dieser Quelle entspringt hauptsächlich die Unsicherheit in den nachfolgenden Rechnungen. Wird das doppelte der Konzentration des komplexen Salzes $\mathrm{KCuCl}_{2}$ von dem Gesamtchlorgehalt abgezogen, so erhält man das in Form freier Ionen oder in Form von undissoziierten $\mathrm{KCl}$ oder $\mathrm{CuCl}$ gelöste Chlor. Die Menge der Chlorionen a wurde berechnet, indem ein durchschnittlicher Dissoziationsgrad $\alpha$ für $\mathrm{CuCl}_{2}$ und $\mathrm{KCl}$ der Rechnung zu Grunde gelegt wurde. Das gesamte Cuprokupfer ergab sich aus der Differenz zwischen Gesamtkupfer und dem maalsanalytisch gefundenen Cuprikupfer. Wurde hiervon das wie oben berechnete $\mathrm{KCuCl}_{2}$ abgezogen, so erhielt man das freie Cuprochlorid und durch Multiplikation mit $\alpha$ die freien Cuproionen $b$. Aus dem Gehalt an Cuprisalz ergiebt sich durch Multiplikation mit $\alpha$ die Menge der Cupriionen $e$. Die folgende Tabelle 7 enthält die auf diesem Wege berechneten Zahlen.

\section{Tabelle 7.}

Löslichkeit von Kupferchlorür in Chlorkaliumlösungen. Millimole im Liter.

\begin{tabular}{r|c|c|c|c|c|c|c}
\hline \hline $\mathrm{Nr}$. & $\begin{array}{c}\text { Konzen- } \\
\text { tration } \\
\text { des } \\
\mathrm{KCl}\end{array}$ & $\begin{array}{c}\text { durch- } \\
\text { schnitlicher } \\
\text { Dissoziations- } \\
\text { grad für die } \\
\text { Chloride } \\
\alpha\end{array}$ & $\begin{array}{c}\text { Gesamt- } \\
\mathrm{KCuCl}_{2}\end{array}$ & $\begin{array}{c}\text { freie } \\
\text { Chlor- } \\
\text { ionen be- } \\
\text { rechnet } \\
\mathrm{a}\end{array}$ & $\begin{array}{c}\text { Freies } \\
\text { CuCl } \\
\text { berechnet }\end{array}$ & $\begin{array}{c}\text { freie } \\
\text { Cupro- } \\
\text { ionen } \\
\mathrm{b}\end{array}$ & $\begin{array}{c}\text { freie } \\
\text { Cupr- } \\
\text { ionen } \\
\text { ch }\end{array}$ \\
\hline \hline 1 & 0 & 0.91 & 0.248 & 4.55 & 0.398 & 0.362 & 2.05 \\
2 & 1 & 0.91 & 0.238 & 4.38 & 0.246 & 0.224 & 1.73 \\
3 & 2 & 0.91 & 0.253 & 4.65 & 0.336 & 0.306 & 1.43 \\
4 & 2.5 & 0.92 & 0.271 & 5.03 & 0.263 & 0.242 & 1.31 \\
5 & 3 & 0.93 & 0.282 & 5.28 & 0.178 & 0.165 & 1.42 \\
6 & 5 & 0.93 & 0.339 & 6.37 & 0.175 & 0.162 & 0.938 \\
7 & 10 & 0.93 & 0.528 & 9.97 & 0.233 & 0.216 & 0.442 \\
8 & 15 & 0.92 & 0.740 & 13.76 & 0.282 & 0.259 & 0.289 \\
9 & 20 & 0.91 & 0.917 & 17.80 & 0.205 & 0.186 & 0.295 \\
10 & 30 & 0.90 & 1.437 & 26.10 & 0.193 & 0.173 & 0.118 \\
11 & 50 & 0.88 & 2.360 & 42.00 & 0.051 & 0.0499 & 0.0957
\end{tabular}

Es sind nur die 11 ersten Reihen der Tabelle 6, in denen noch Cuprichlorid in mefsbaren Mengen vorhanden war, berücksichtigt worden.

Die Tabelle 8 enthält die Bezeichnung der Gröfsen, welche in dem einen oder dem anderen Falle hätten konstant sein müssen. 
Tabelle 8.

Löslichkeit von Kupferchlorür in Chlorkaliumlösungen.

Fortsetzung von Tabelle 6 und 7 .

\begin{tabular}{|c|c|c|c|c|c|c|c|c|c|c|}
\hline $\mathrm{Nr}$. & $\frac{b}{c}$ & 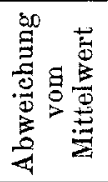 & $\frac{b^{2}}{e}$ & 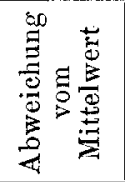 & c. $a^{2}$ & 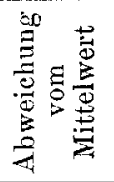 & b.a & 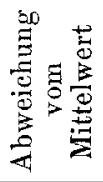 & b. $\mathrm{a}^{2}$ & 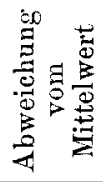 \\
\hline 1 & 0.176 & +0.274 & 0.0640 & +0.0254 & 42.5 & +19.4 & 1.65 & +0.42 & 7.51 & +25.66 \\
\hline 2 & 0.129 & +0.321 & 0.0290 & +0.0604 & 33.1 & +28.8 & 0.98 & +1.09 & 4.29 & +28.88 \\
\hline 3 & 0.214 & +0.236 & 0.0654 & +0.0240 & 30.9 & +31.0 & 1.42 & +0.65 & 6.60 & +26.57 \\
\hline 4 & 0.185 & +0.265 & 0.0448 & +0.0446 & 33.1 & +28.8 & 1.22 & +0.85 & 6.13 & +27.04 \\
\hline 5 & 0.117 & $\begin{array}{r}+0.333 \\
\end{array}$ & 0.0194 & +0.0700 & 39.5 & +22.4 & 0.87 & +1.19 & 4.62 & +28.55 \\
\hline 6 & 0.173 & +0.277 & 0.0282 & +0.0612 & 60.3 & $+\quad 1.6$ & 1.04 & +1.03 & 6.60 & +26.57 \\
\hline 7 & 0.490 & -0.040 & 0.1060 & -0.0166 & 43.9 & +18.0 & 2.26 & -0.19 & 22.60 & +10.57 \\
\hline 8 & 0.897 & -0.447 & 0.2320 & -0.1426 & 54.7 & $\begin{array}{r}+\quad 7.2 \\
\end{array}$ & 3.57 & -1.50 & 49.10 & -15.93 \\
\hline 9 & 0.633 & -0.183 & 0.1180 & -0.0286 & 93.3 & -31.4 & 3.32 & -1.25 & 59.10 & -25.93 \\
\hline 10 & 1.470 & -1.020 & 0.2560 & -0.1666 & 80.4 & -18.5 & 4.54 & -2.47 & 1.19 .00 & -85.83 \\
\hline 11 & 0.469 & -0.019 & 0.0211 & +0.0683 & 169.0 & -107.1 & 1.88 & +0.19 & 79.30 & -46.13 \\
\hline $\begin{array}{c}\text { Mittel- } \\
\text { wert }\end{array}$ & 0.450 & \pm 0.310 & 0.0894 & \pm 0.0644 & 61.9 & +28.6 & 2.07 & \pm 0.98 & 33.17 & \pm 31.60 \\
\hline
\end{tabular}

Die Resultate sind keineswegs befriedigend. Unabhängig von jeder Hypothese sollte die Beziehung gelten $\left(\mathrm{Cu}^{*}\right)\left(\mathrm{Cl}^{\prime}\right)^{2}=c a^{2}=$ konstant. Es beträgt aber die mittlere Abweichung dieser Werte vom Mittelwert 46.1\%. Die gröfste Abweichung zeigen die Versuche mit hohem Chlorkaliumgehalt. Das liegt sehr wahrscheinlich daran, dafs bei Gegenwart von viel Chlorionen die Dissoziation des Cuprichlorids nicht einfach nach dem Schema $\mathrm{CuCl}_{2}=\mathrm{Cu}+2 \mathrm{Cl}$ erfolgt, sondern dals daneben in beträchlicher Menge Ionen $\mathrm{CuCl}^{-}, \mathrm{CuCl}_{3}{ }^{\prime}$ oder andere auftreten. Unsere Kenntnis der Menge der $\mathrm{Cu}^{*}$-Ionen ist deshalb sehr unsicher.

Die Unsicherheit der Werte von $c$ beeinflufst naturgemäls die Werte $\frac{b}{c}$ und $\frac{b^{2}}{c}$; es kommt hinzu die grolse Unsicherheit in den experimentellen und theoretischen Grundlagen der Berechnung der freien Cuproionen. Schon das Cuprokupfer ergiebt sich aus der Differenz zweier direkt bestimmter Grölsen und ist deshalb mit den Fehlern beider Bestimmungsarten behaftet. Hiervon mufs wieder das in Form der komplexen Verbindung vorhandene Cuprokupfer abgezogen werden, dessen Bestimmung gleichfalls ungenau ist, und es mufs der Einflufs des ungenau bekamnten Dissoziationsgrades berücksichtigt werden. Die Abwcichungen 
der berechneten Werte rom Mittelwert betragen durchschnittlich 69 bezw. $72 \%$. Eine Entscheidung, welcher von beiden Ausdrücken weniger von der Konstanz entfernt ist, kann demnach nicht getroffen werden.

Etwas günstiger liegen die Verhältnisse für die Berechnung der Ausdrücke $b . a$ bezw. $b . a^{2}$. Die Menge $a$ der freien Chlorionen ist voraussichtlich nicht mit sehr grofsen Fehlern behaftet und nur die Unsicherheit in den Werten von $b$, d. h. der Menge der freien Cuproionen bleibt bestehen. Die mittlere Abweichung vom Durchschnittswert beträgt für den Ausdruck $b . a 47.5 \%$ für den Ausdruck $a . b^{2}$ $95.3 \%$.

Wenn man aus Berechnungen, die mit so grolsen experimentellen und theoretischen Fehlern behaftet sind, einen Schlufs ziehen darf, so wäre es der, dals eher der Ausdruck b.a als $b . a^{2}$ konstant ist, dals also die freien Cuproionen einatomig sind.

Der vorläufigen Mitteilung ${ }^{1}$ lagen nur einzelne von den hier angeführten Versuchen zu Grunde, welche die Annahme einatomiger Cuproionen besser begründet erscheinen liefsen. Andere Versuche, deren Deutung voraussichtlich geringeren theoretischen Schwierigkeiten begegnen wird, sind zur Erscheinung der Frage nach der Wertigkeiten der freien Cuproionen in Angriff genommen; sie betreffen die Löslichkeit des Cuprobromids bei Gegenwart von Bromkalium.

Bevor die Frage nach der Formel der freien Cuproionen entschieden ist, soll auf die Frage nach ihrer Haftintensität und nach der freien Energie des Überganges der Cupro- in die Cupriionen nicht eingegangen werden.

\section{Messungen von Konzentrationsketten.}

Es war oben gezeigt worden, dafs die Löslichkeit des Kupferchlorürs in Chloridlösungen verschiedener Konzentration nur die Entscheidung liefert, dafs in Lösungen von geringerem Chlorkaliumgehalt Moleküle der Formel $(\mathrm{CuCl})_{n} \mathrm{KCl}$, in solchen von höherem Chlorkaliumgehalt Moleküle der Formel $(\mathrm{CuCl})_{m} \mathrm{~K}_{2} \mathrm{Cl}_{2}$ enthalten sind. Dagegen lärst sich auf diesem Wege nicht feststellen, welchen Wert $n$ und $m$ besitzen. Die Entscheidung dieser Frage ist durch Messungen von Konzentrationsketten möglich. ${ }^{2}$

1 Chem.Ztg. 24 (1900), 82. Versammlg. deutscher Naturf. u. Ärzte in Aachen, Sitzung für Chemie, Vortrag v. Bodländer.

2 Vergl. G. BoduäNDER. Die Untersuchung von komplexen Verbindungen. Aus der Festschrift für Rtchard Dedekind. Braunschweig 1901. S. 153-182. 
Wenn die Verbindungen die Formel $\mathrm{KCuCl}$, ihre Anionen also die Formel $\mathrm{CuCl}_{2}^{\prime}$ besitzen, so sind diese ihrerseits wieder in geringem Umfange in Cuproionen und Chlorionen gespalten:

Es ist also:

$$
\mathrm{CuCl}_{2}{ }^{\prime}=\mathrm{Cu}+2 \mathrm{Cl} \text {. }
$$

$$
\frac{\left(\mathrm{Cu}^{\circ}\right)\left(\mathrm{Cl}^{\prime}\right)^{2}}{\left(\mathrm{CuCl}_{2}^{\prime}\right)}=k
$$

wo die Klammerausdrücke die Konzentration der Ionenarten bedeuten.

Wenn wir in zwei Gläser $a$ und $b$ dieselbe mit Cuprochlorid nicht gesättigte Lösung von Kaliumcuprochlorid bringen, die einen sehr beträchtlichen Überschufs von Chlorkalium entbält, und zu der Lösung in dem einen Gefäls noch eine Lösung von Chlorkalium, welche dieselbe Konzentration besitzt, wie diejenige, die zur Herstellung der Kaliumcuprochloridlösung gedient hatte, so ist der Gehalt an Chlorionen in beiden Gefälsen nahe derselbe; dagegen enthält das Gefäls $a$ infolge der Verdünnung durch die Chlorkaliumlösung weniger Kaliumeuprochlorid.

Wenn die Verdünnung in dem Verhältnis $1: n$ erfolgt, so ist $\left(\mathrm{CuCl}_{2}{ }^{\prime}\right)$ im Gefäls $b$ mal so grols als im Gefäls $a$, also $\left(\mathrm{CuCl}_{2 b}{ }^{\prime}\right)$ $=n\left(\mathrm{CuCl}_{2}{ }^{\prime}\right)$. Nun ist:

$$
\frac{\left(\mathrm{Cu}_{a}{ }^{\circ}\right) \cdot\left(\mathrm{Cl}_{a}{ }^{\prime}\right)^{2}}{\left(\mathrm{CuCl}_{2 a^{a}}{ }^{\prime}\right)}=\frac{\left(\mathrm{Cu}_{b}{ }^{\circ}\right) \cdot\left(\mathrm{Cl}_{b}{ }^{\prime}\right)^{2}}{\left(\mathrm{CuCl}_{2 b}{ }^{\prime}\right)}=k,
$$

$\left(\mathrm{Cl}_{a}{ }^{\prime}\right)$ ist gleich $\left(\mathrm{Cl}_{b}{ }^{\prime}\right)$, mithin

$$
\frac{\left(\mathrm{Cu}_{a}{ }^{\circ}\right)}{\left(\mathrm{Cu}_{b}{ }^{\circ}\right)}=\frac{\left(\mathrm{CuCl}_{2}{ }^{\prime}\right)}{\left(\mathrm{CuCl}_{2^{b}}{ }^{\prime}\right)}=\frac{1}{n} .
$$

Befindet sich in beiden Gefälsen eine Kupferelektrode und sind die Gefäfse durch einen die Chlorkaliumlösung enthaltenden Heber verbunden, so erhalten wir eine Konzentrationskette.

Es wird das Kupfer in dem Gefäls $b$ positiver $\mathrm{Pol}$ und die elektromotorische Kraft der Konzentrationskette ist, da die elektromotorische Kraft der Flüssigkeitskette $=0$ ist,

$$
E=0.058 \log n .
$$

Wenn die Formel des komplexen Salzes $\mathrm{K}_{2} \mathrm{CuCl}_{3}$ ist, die der Anionen also $\mathrm{CuCl}_{3}{ }^{\prime \prime}$, so ist: 


$$
\begin{gathered}
-29- \\
\mathrm{CuCl}_{3}{ }^{\prime \prime}=\mathrm{Cu}^{\circ}+3 \mathrm{Cl}^{\prime} \text { und } \\
\frac{\left(\mathrm{Cu}^{\prime}\right)\left(\mathrm{Cl}^{\prime}\right)^{3}}{\left(\mathrm{CuCl}_{3}^{\prime \prime}\right)}=k .
\end{gathered}
$$

Da auch hier wieder die Konzentration der Chlorionen in beiden Fällen dieselbe ist, die Menge des komplexen Salzes in $b$ aber $n$ mal so grofs als in $a$, so ist:

$$
\frac{\left(\mathrm{Cu}_{a}{ }^{\circ}\right)}{\left(\mathrm{Cu}_{b}{ }^{\circ}\right)}=\frac{\left(\mathrm{CuCl}_{3 a^{\prime \prime}}\right)}{\left(\mathrm{CuCl}_{3 b^{\prime \prime}}\right)}=-\frac{1}{n} .
$$

Die elektromotorische Kraft der Konzentrationskette ist also auch hier:

$$
E=0.058 \log n .
$$

Es lärst sich also durch die Messung dieser elektromotorischen Kräfte nicht entscheiden, ob das komplexe Salz die Formel $\mathrm{KCuCl}_{2}$ oder $\mathrm{K}_{2} \mathrm{CuCl}_{3}$ oder $\mathrm{K}_{3} \mathrm{CuCl}_{4}$ u. s. w. besitzt.

Wenn aber die Formel des komplexen Salzes $\mathrm{KCu}_{2} \mathrm{Cl}_{3}$ ist, wenn es also entsteht, indem 1 Molekül Chlorkalium ein Doppelmolekül $\mathrm{Cu}_{2} \mathrm{Cl}_{2}$ addiert, so wird ein sehr kleiner Teil der komplexen Anionen nach der Gleichung zerfallen:

$$
\mathrm{Cu}_{2} \mathrm{Cl}_{3}{ }^{\prime}=2 \mathrm{Cu}^{\cdot}+3 \mathrm{Cl}^{\prime}
$$

es ist mithin:

$$
\frac{\left(\mathrm{Cu}^{\circ}\right)^{2} \cdot\left(\mathrm{Cl}^{\prime}\right)^{3}}{\left(\mathrm{Cu}_{2} \mathrm{Cl}_{3}{ }^{\prime}\right)}=k
$$

auch hier ist in den beiden Gefälsen ( $\left.\mathrm{Cl}^{\prime}\right)$ konstant. Dagegen ist:

$$
\begin{gathered}
\left(\mathrm{Cu}_{2} \mathrm{Cl}_{3 a^{\prime}}\right):\left(\mathrm{Cu}_{2} \mathrm{Cl}_{3^{b}}\right)=\frac{1}{n}, \text { mithin: } \\
\frac{\left(\mathrm{Cu}_{a}\right)^{2}}{\left(\mathrm{Cu}_{b}\right)^{2}}=\frac{\left(\mathrm{Cu}_{2} \mathrm{Cl}_{3^{\prime}}\right)}{\left(\mathrm{Cu}_{3} \mathrm{Cl}_{3^{\prime}}{ }^{\prime}\right)}=\frac{1}{n}, \\
\frac{\left(\mathrm{Cu}_{a}{ }^{\circ}\right)}{\left(\mathrm{Cu}_{b^{\circ}}\right)}=\sqrt{\frac{1}{n}} \cdot
\end{gathered}
$$

Wenn die freien Cuproionen einatomig sind, so ist die elektromotorische Kraft der Konzentrationskette

$$
E=0.058 \log \sqrt{n}=0.029 \log n .
$$


Das Gleiche ergiebt sich, wenn die Formel des komplexen Salzes $\mathrm{K}_{2} \mathrm{CuCl}_{4}$ oder $\mathrm{K}_{3} \mathrm{CuCl}_{5}$ ist, d. h. immer dann, wenn $1,2,3$ $n$-Moleküle Chlorkalium sich mit einem Doppelmolekül $\mathrm{Cu}_{2} \mathrm{Cl}_{2}$ vereinigen. Auch wenn die einfachen Cuproionen nicht ein-, sondern zweiatomig sind, erhalten wir dieselben Werte für die Konzentrationsketten.

Ist die Formel des komplexen Salzes $\mathrm{KCuCl}_{2}$, die der freien Cuproionen aber $\mathrm{Cu}_{2}{ }^{*}$, so gilt die Beziehung:

$$
\begin{gathered}
2 \mathrm{CuCl}_{2}{ }^{\prime}=\mathrm{Cu}_{2}{ }^{*}+\mathrm{Cl}^{\prime}, \\
\frac{\left(\mathrm{Cu}_{2}{ }^{\prime}\right)\left(\mathrm{Cl}^{\prime}\right)^{4}}{\left.\left(\mathrm{CuCl}_{2}\right)^{2}\right)^{2}}=k .
\end{gathered}
$$

Wir erbalten wieder bei gleicher Anordnung wie oben eine Konzentrationskette. Die Konzentration der Chlorionen ist in $a$ und $b$ dieselbe, die Konzentration der Ionen $\mathrm{CuCl}_{2}{ }^{\prime}$ ist in $b \quad n$ mal so grofs, wie in $a$; nun ist:

$$
\frac{\left(\mathrm{Cu}_{2^{a}} a^{\prime \prime}\right)}{\left(\mathrm{Cu}_{2^{b}} \cdot\right)}=\frac{\left(\mathrm{CuCl}_{2 a}\right)^{2}}{\left(\mathrm{CuCl}_{2^{b}}\right)^{2}}=\left(-\frac{1}{n}\right)^{2} .
$$

Da die freien Cuproionen zweiwertig sind, so ist die elektromotorische Kraft der Konzentrationskette:

$$
E=0.029 \log n^{2}=0.058 \log n,
$$

sie ist also ebenso grols, wie wenn die freien Cuproionen einatomig wären. Über die Atomigkeit der freien Cuproionen giebt́ diese Konzentrationskette mithin keinen Aufschlufs.

Dasselbe ergiebt sich auch, wenn man annimmt, dafs das komplexe Salz vom Typus $\mathrm{KCu}_{2} \mathrm{Cl}_{3}$ oder $\mathrm{K}_{2} \mathrm{Cu}_{2} \mathrm{Cl}_{4}$ u. s. w. ist. Die elektromotorische Kraft ist dann, auch wenn die freien Cuproionen zweiatomig sind, $0.029 \log n$ Volt.

Wir erfahren aus diesen Konzentrationsketten nichts über die Formel der einfachen Cuproionen und auch nichts über die Anzahl der Moleküle Chlorkalium, die sich mit einem Molekül Cuprochlorid vereinigen. Wohl aber giebt uns die Konzentrationskette darüber Aufschlufs, ob ein Molekül der komplexen Verbindung ein, zwei oder drei Atome Kupfer enthält.

Es war notwendig, bei diesen Messungen die Oxydation der Lösungen durch den Luftsauerstoff vollständig auszuschliefsen. Dies wurde erreicht, indem den Zellen, in denen die Messungen erfolgten, 
die Form Fig. 3 gegeben wurde. Sie bestanden aus zwei weithalsigen Glasflaschen von ca. $200 \mathrm{ccm}$ Inhalt, die durch dreifach durchbohrte Gummistopfen verschlossen waren. Sie trugen je ein bis auf den Boden reichendes Glasrohr $l$ und ein kurzes Glasrohr k, die mit Schläuchen und Quetschhähnen verschliefsbar waren, sowie ein mit Dreiweghahn versehenes Heberrohr $h$. Aufserdem waren in ihnen die elektrolytisch verkupferten Kupferelektroden $e$ gasdicht

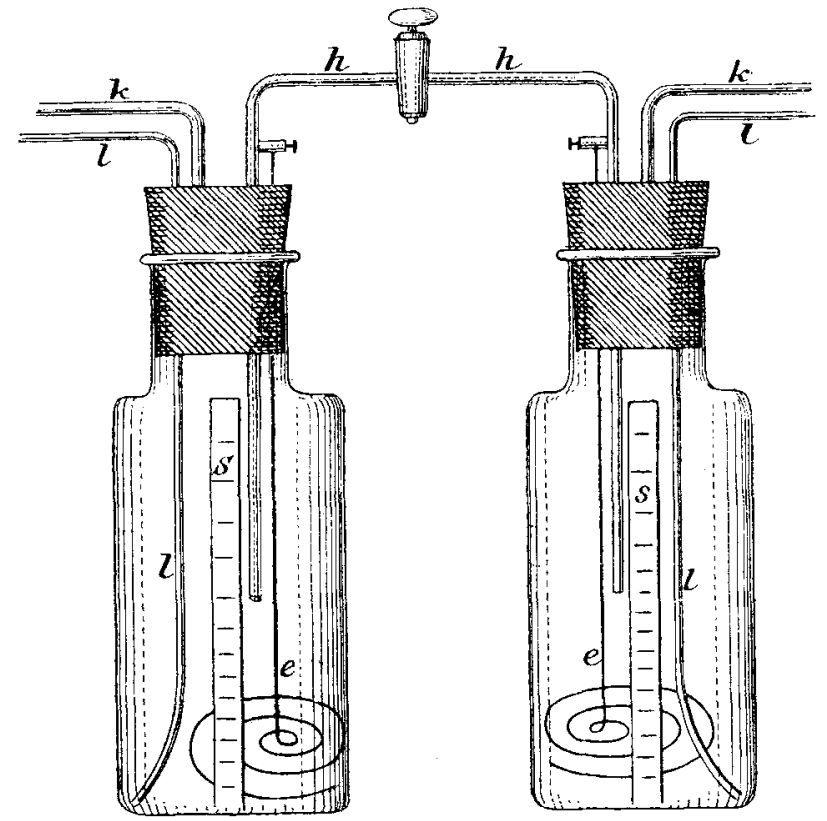

Fig. 3.

befestigt. Aufsen war eine Papierskala $s$ angebracht, die die Flüssigkeit in den Gefälsen abzumessen gestattete.

Vor der Benutzung wurden beide Gefälse mit Kohlensäure gefüllt. Dann wurde in ein Gefäls aus dem früher beschriebenen Kolben die mit Kupferchlorür nahezu gesättigte Lösung filtriert, indem das Filterrohr mit $l$ verbunden wurde, während die Kohlensäure durch $k$ und ein Wasserventil austrat. Die Kupferchlorürlösung wurde dann mit einer gemessenen Menge derselben lufttreien Chlorkaliumlösung verdünnt, welche zur Sättigung mit Kupferchlorür verwendet worden war, so dafs die Lösung jetzt für Kupferchlorür sicher untersättigt war. Sie wurde durch Kohlensäuredruck, der von $k$ aus wirkte, durch die Röhren $l$ und zwischengeschobene Glas- 
und Kautschukverbindungen aus dem einen Gefäls in das zweite geprefst, dann wieder zurück und schliefslich in beiden Gefäfsen gleichmäfsig verteilt, wobei der Heber $h$ mit der Flüssigkeit gleichfalls gefüllt wurde. Es wurde ein etwaiger Spannungsunterschied zwischen den Elektroden, der meist kleiner als 2 Millivolt war, festgestellt, und zur Korrektur der Messungen verwendet. Dann wurde die Lösung in dem einen Gefäfs unverändert gelassen; die andere aber wurde mit der kupferfreien Chlorkaliumlösung verdünnt, so dals jetzt der Chlorkaliumgehalt an beiden Elektroden der gleiche, der Kupfergehalt ein verschiedener war. Die Messungen der elektromotorischen Kraft erfolgten mittelst des von BODLÄNDER und BREULL ${ }^{3}$ beschriebenen Apparates nach dem Kompensationsverfahren. Der Hahn des Heberrohrs blieb dabei meist geschlossen, da die den Küken benetzende Flüssigkeit genügend gut leitete.

Ein Versuch, in dem der Chlorkaliumgehalt beider Lösungen 0.1 war, die Kupfergehalte aber im Verhältnis 1:4 standen, ergab eine elektromotorische Kraft von 0.0351 Volt.

Die elektromotorische Kraft dieser Zelle ist nicht ausschliefslich durch die Verschiedenheit im Kupfergehalt der beiden Lösungen bedingt, sondern zu einem kleinen Teil auch durch die Verschiedenheit des Chlorgehaltes. Zwar waren die angewandten Chlorkaliumlösungen identisch; von dem freien Chlorkalium den Chlorionen ist aber ein Teil durch Bildung der komplexen Ionen verbraucht worden. Es addiert, wie die nachfolgenden Messungen ergaben, jedes Äquivalent Cuprochlorid ein Molekül Chlorkalium in Lösungen, die für Chlorkalium etwa 0.1 normal sind. Die Lösung in Zelle $b$ war für Kupfer 0.004 normal, die Lösung in a 0.001, folglich war der Gehalt an freiem Chlorkalium in $b$ nicht 0.1 , sondern nur 0.096 , in $a=0.099$. Dieser Unterschied im Chlorgehalt bedingt, wie weiter unten gezeigt wird, eine elektromotorische Kraft von der Grölse $0.058 \log \left(\frac{0.099}{0.096}\right)^{2}=0.0015$ Volt von demselben Vorzeichen wie diejenige, die durch den Unterschied der Kupferkonzentration bedingt ist. Letztere ist also $0.0351-0.0015=0.0336$.

Wenn die Lösung das Kupfer in Form des Salzes $\mathrm{K}_{n} \mathrm{CuCl}_{n+1}$ enthielt, so mulste die elektromotorische Kraft der Konzentrationskette sein:

Die Bildung des Natriumbikarbonats. Zeitschr. angew. Chem. 14 (1901), 409 . 


$$
\begin{gathered}
-33- \\
E=0.058 \log 4=0.0348,
\end{gathered}
$$

während sich die elektromotorische Kraft:

$$
E=0.029 \log 4=0.0174
$$

ergeben mufste, wenn die Formel des komplexen Salzes $\mathrm{K}_{n} \mathrm{Cu}_{2} \mathrm{Cl}_{n+2}$ wäre.

Die nahe Übereinstimmung des gefundenen Wertes $0.0336 \mathrm{mit}$ dem theoretischen Werte 0.0348 beweist, dafs nur 1 Atom Kupfer in dem Molekül des komplexen Salzes vorhanden ist, ohne über die Anzahl der Kalium- und Chloratome Aufschlufs zu geben.

Eine kleinere elektromotorische Kraft ergab sich in Versuchen, in denen die Chlorkaliumkonzentration in beiden Zellen nur 0.05 normal war. Bei dem Kupferverhältnis von 1:4 ergab sich die elektromotorische Kraft 0.0229 Volt statt 0.0348 . Indessen ist diese Abweichung nur eine scheinbare. In den 0.2 und 0.1 norm. Lösungen lälst sich durch die Reaktion mit Jodkalium die Gegenwart der Cupriverbindung nicht mehr feststellen, dagegen enthält die Lösung von Cuprochlorid in 0.05 normaler Chlorkaliumlösung $0.0001088 \mathrm{mg}$ Moleküle $\mathrm{CuCl}_{2}$. Wäre das gesamte Kupfer in Form von Cupriionen vorhanden, so mürsten diese bei der Verdünnung der Lösung auf das vierfache eine elektromotorische Kraft von $0.029 \log 4=0.0174$ ergeben, da die Cupriionen zweiwertig sind. Wären dagegen gar keine Cuprïionen und nur freie Cuproionen vorhanden, so wäre die elektromotorische Kraft wie oben $0.058 \log 4=0.0348$ Volt; dafs der gefundene Wert in der Mitte liegt, bestätigt, was die quantitative Untersuchung der Lösung ergeben hatte, dafs Kupfer zum Teil als Cuprichlorid bezw. Cupriionen, zum Teil in Form des komplexen Salzes $\mathrm{K}_{n} \mathrm{CuCl}_{n+1}$ bezw. seiner Ionen zum Teil in Form freier Cuproionen vorhanden ist.

Um zu ermitteln, in welcher Form das Kupfer in den konzentrierteren Lösungen vorhanden ist, wurde auch ein Versuch mit normaler Chlorkaliumlösung unternommen. Es wurden in der oben beschriebenen Weise zwei Lösungen verglichen, die für Chlorkalium 1 normal waren. Lösung $a$ enthielt 0.0162 Grammmoleküle Cuprochlorid (als $\mathrm{CuCl}$ berechnet), Lösung $b 0.0485$ Grammmoleküle.

Die Löslichkeitsversuche S. 18 ergaben, dals in normaler Lösung zur Bildung des komplexen Salzes für ein Molekül $(\mathrm{CuCl})_{n}$ zwei Moleküle Chlorkalium verbraucht werden. Nun ist unbekannt und soll erst ermittelt werden, ob das komplexe Salz die Formel $\mathrm{CuCl}_{3} \mathrm{~K}_{2}$ 
oder $\mathrm{Cu}_{2} \mathrm{Cl}_{4} \mathrm{~K}_{2}$ besitzt. Im ersten Falle würden zur Bildung des komplexen Salzes in Lösungen $2 \times 0.0162=0.0324$ Grammmoleküle Chlorkalium verbraucht werden, die Lösung enthielte also an freiem Chlorkalium uur noch 0.9676 Grammmoleküle Chlorkalium, die Lösung $b$ enthält unter derselben Annahme $1-2 \times 0.0485=0.9030$ Grammmoleküle Chlorkalium. Es sind also die lösungen für Chlır nicht mehr gleich konzentriert. Wenn die Formel der komplexen Ionen $\mathrm{CuCl}_{3}{ }^{\prime \prime}$ ist, besteht die Gleichung:

$$
\begin{gathered}
\mathrm{CuCl}_{3}{ }^{\prime \prime}=3 \mathrm{Cl}^{\prime}+\mathrm{Cu}^{\circ} \\
k\left(\mathrm{CuCl}_{3}\right)=(\mathrm{Cl})^{3} \cdot(\mathrm{Cu}) .
\end{gathered}
$$

Daraus ergiebt sich für den Gehalt der beiden Lösungen an Cuproionen $(\mathrm{Cu})$ und $(\mathrm{Cu})_{1}$ die Beziehung:

$$
\frac{(\mathrm{Cu})}{(\mathrm{Cu})_{1}}=\frac{\left(\mathrm{CuCl}_{3}\right)}{\left(\mathrm{CuCl}_{3}\right)_{1}} \cdot \frac{(\mathrm{Cl})_{1}^{3}}{(\mathrm{Cl})^{3}}
$$

Die elektromotorische Kraft mülste dann sein:

$$
E=0.058 \log \frac{\left(\mathrm{CuCl}_{3}\right)}{\left(\mathrm{CuCl}_{3}\right)_{1}} \cdot \frac{(\mathrm{Cl})_{1}^{3}}{(\mathrm{Cl})^{3}}
$$

Wir könnten mit einiger Annäherung annehmen, dals das komplexe Salz ebenso wie das Chlorkalium in beiden Lösungen gleich stark dissoziiert ist und für die Konzentration der Ionen die der Salze selbst einsetzen. Der berechnete Wert wäre dann:

$$
E=0.058 \log \frac{0.0486 \cdot(0.9676)^{3}}{0.0162 \cdot(0.9030)^{3}}=0.0328
$$

Gefunden wurde 0.0285 Volt.

Ist aber die Formel des komplexen Salzes $\mathrm{K}_{2} \mathrm{Cu}_{2} \mathrm{Cl}_{4}$, so bindet ein Atom Kupfer nur ein Atom Chlorkalium, und der Gehalt an Chlorkalium in beiden Lösungen ist 0.9838 und 0.9515 . Für die elektromotorische Kraft der Konzentrationskette erhält man dann:

$$
E=0.029 \log \frac{0.0486 \cdot(0.9838)^{3}}{0.0162 \cdot(0.9515)^{3}}=0.0151 \text {. }
$$

Der Versuch spricht zweifellos zu Gunsten der ersten Annahme, dals also auch in den komplexen Salzen, die in konzentrierteren Lösungen vorhanden sind, nur ein Atom Kupfer im Molekül vorhanden ist. 
In Kombination mit den Ergebnissen der Löslichkeitsversuche folgt für das komplexe Salz die Formel:

$$
\mathrm{K}_{2} \mathrm{CuCl}_{3} \text {. }
$$

Dieser Schlufs wird auch durch die Messung der elektromotorischen Kräfte der Ketten der zweiten Art bestätigt.

\section{Konzentrationsketten bei verschiedenem Chlorkaliumgehalt.}

Durch Messung der Konzentrationsketten war nur über die Anzahl der Kupferatome im Molekül und den Ionen des Kaliumcuprochlorids Aufschlufs gewonnen worden und es hatte sich ergeben, dafs zwischen Chlorkaliumkonzentrationen von $0.05-2$ normal immer nur ein Atom Kupfer in dem Salz vorhanden ist. Unentschieden bleibt es, wie viel Moleküle Chlorkalium mit einem Molekül Cuprochlorid verbunden sind, ob also das komplexe Salz die Formel $\mathrm{KCuCl}_{2}$ oder $\mathrm{K}_{2} \mathrm{CuCl}_{3}$ oder $\mathrm{K}_{n} \mathrm{CuCl}_{n+1}$ besitzt. Auch darüber kann Aufschlufs gewonnen werden, wenn man die Konzentrationsketten von Chlorkaliumlösungen mifst, die das komplexe Salz enthalten, ohne mit Cuprochlorid gesättigt zu sein; doch mufs hierbei die Chlorkonzentration in den beiden Zellen des Elementes verschieden sein, während die Kupferkonzentration zweckmälsig gleich gemacht wird.

Wenn das komplexe Salz die Formel $\mathrm{K}_{m} \mathrm{Cu}_{n} \mathrm{Cl}_{m+n}$, seine Anionen also die Formel $\mathrm{Cu}_{n} \mathrm{Cl}_{m+n}$ besitzen, so unterliegen diese einer teilweisen Dissoziation nach der Gleichung:

$$
\mathrm{Cu}_{n} \mathrm{Cl}_{m+n}=n \mathrm{Cu}+(m+n) \cdot \mathrm{Cl},
$$

woraus sich die Gleichgewichtsbedingung ergiebt:

$$
\frac{(\mathrm{Cu})^{n} \cdot(\mathrm{Cl})^{m+n}}{\left(\mathrm{Cu}_{n} \mathrm{Cl}_{m+n}\right)}=k \text {. }
$$

Ist die Kupferkonzentration in zwei Lösungen identisch, so können wir in erster Annäherung auch die Konzentration der Anionen $\mathrm{Cu}_{n} \mathrm{Cl}_{m+n}$ für gleich ansehen. Bei verschiedenem Chlorkaliumgehalt der beiden Lösungen ist allerdings der Dissoziationsgrad des komplexen Salzes etwas verschieden, so dafs bei gleichem Gesamtkupfergehalt die Mengen der Anionen in der an Chlorkalium konzentrierten Lösung etwas kleiner sein wird. Nehmen wir aber, was 
in erster Annäherung zutreffen wird, an, dals die Menge der Anionen $\mathrm{Cu}_{n} \mathrm{Cl}_{m+n}$ praktisch gleich ist, so ergiebt sich für die Konzentration der Cuproionen in den Lösungen $a$ und $b$ die Bedingung:

$$
\frac{\left(\mathrm{Cu}_{a}\right)^{n}}{\left(\mathrm{Cu}_{b}\right)^{n}}=\frac{\left(\mathrm{Cl}_{b}\right)^{m+n}}{\left(\mathrm{Cl}_{a}\right)^{m+n}} \text { oder } \frac{\left(\mathrm{Cu}_{a}\right)}{\left(\mathrm{Cu}_{b}\right)}=\left[\begin{array}{l}
\left(\mathrm{Cl}_{b}\right) \\
\left(\mathrm{Cl}_{a}\right)
\end{array}\right]^{m+n}
$$

sind die freien Cuproionen einatomig, so ist bei Anwendung von Kupferelektroden die elektromotorische Kraft der Konzentrationskette:

$$
E=0.058 \log \frac{\left(\mathrm{Cu}_{a}\right)}{\left(\mathrm{Cu}_{b}\right)}=\left(\frac{m+n}{n}\right) 0.058 \log \frac{\left(\mathrm{Cl}_{b}\right)}{\left(\mathrm{Cl}_{a}\right)}
$$

Dieselbe Formel ergiebt sich auch, wenn die Cuproionen nicht einatomig, sondern zwei- oder mehratomig sind. Sind sie nämlich zweiatomig, so erfolgt der Zerfall der komplexen Anionen nach der Gleichung:

$$
\mathrm{Cu}_{n} \mathrm{Cl}_{m+n}=\frac{n}{2} \mathrm{Cu}_{2}+(m+n) \mathrm{Cl},
$$

woraus sich die Gleichgewichtsbedingung ergiebt:

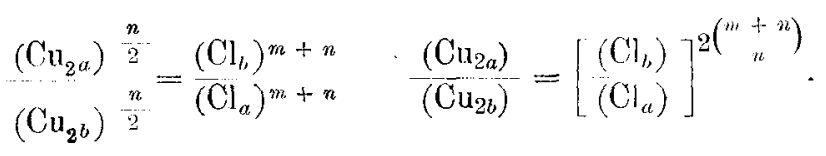

Da die Cuproionen als zweiatomig angenommen werden, ist die elektromotorische Kraft der Konzentrationskette:

$$
\begin{gathered}
E=0.029 \log \frac{\left(\mathrm{Cu}_{2 a}\right)}{\left(\mathrm{Cu}_{2 b}\right)}=2\left(\frac{m+n}{n}\right) 0.029 \log \frac{\left(\mathrm{Cl}_{b}\right)}{\left(\mathrm{Cl}_{a}\right)} \\
=\left(\frac{m+n}{n}\right) 0.058 \log \frac{\left(\mathrm{Cl}_{b}\right)}{\left(\mathrm{Cl}_{a}\right)}
\end{gathered}
$$

Die Formel ist also identisch mit der obigen, so dafs auch hier die Messung der elektromotorischen Kraft nichts über die Anzahl der Kupferatome in den freien Cuproionen ergiebt. Es wird ferner auch nicht direkt die Zahl $m+n$ der Chloratome in den komplexen Anionen gefunden, sondern nur das Verhältnis $\frac{m+n}{n}$. Da aber die Konzentrationsketten bei wechselndem Kupfergehalt und gleichem 
Chlorgehalt der Lösung zweifellos ergeben hatten, dals $n=1$ ist, so erhält man direkt die Anzahl der Chloratome in den komplexen Anionen. Die Art, wie diese Konzentrationsketten gemessen wurden, ergiebt sich aus folgendem Beispiel:

Die Anordnung der Zellen war dieselbe wie bei den oben beschriebenen Messungen; es wurden in eine Zelle $20 \mathrm{ccm} 0.1$ normaler luftfreier Chlorkaliumlösung gegeben und dazu $120 \mathrm{ccm}$ einer nahezu gesättigten Lösung von Cuprochlorid in 0.1 normaler Chlorkaliumlösung filtriert. Die gat durchgeschüttelte Mischung wurde zur Hälfte in die zweite Zelle hinübergetrieben. Die von Verschiedenheiten der Elektroden herrührende elektromotorische Kraft betrug 0.0019 Volt und zwar war das Kupfer in Zelle $a$ positiv. Es wurden nun zu den $70 \mathrm{ccm}$ in $a 30 \mathrm{ccm}$ einer luftfreien 0.5 normalen Chlorkaliumlösung und zu den $70 \mathrm{ccm}$ in $b 30 \mathrm{ccm}$ einer luftfreien 0.1 normalen Chlorkaliumlösung hinzugefügt. Der Kupfergehalt beider Zellen war also jetzt identisch; der Chlorkaliumgehalt in $a$ war $0.01(70 \cdot 0.1+30 \cdot 0.5)=0.22$ normal, während der Chlorkaliumgehalt in $b \quad 0.1$ normal war. Bei der Messung erwies sich das Kupfer in $a$ gegen $b$ negativ und zwar betrug die elektromotorische Kraft 0.0355 . Korrigieren wir für die ursprüngliche Potentialdifferenz der beiden Kupferelektroden, so ergiebt sich die elektromotorische Kraft zu 0.0374 .

Den Wert $\left(\frac{m+n}{n}\right)$ erhalten wir aus obiger Gleichung:

$$
\left(\begin{array}{c}
m+n \\
n
\end{array}\right)=\frac{0.0374}{0.058 \log \frac{\mathrm{Cl}_{a}}{\overline{\mathrm{C}_{b}}}}=\frac{0.0374}{0.058 \log 2.2}=1.9 \text {. }
$$

Man kann den wahren Wert in Anbetracht der mannigfachen Fehlerquellen unbedenklich gleich 2 ansetzen; da $n=1$ ist, so ergiebt sich für $m$ der Wert 1 und es hat somit das komplexe Salz in Lösungen, die für Chlorkalium 0.1-0.22 normal sind, die Formel:

$$
\mathrm{KCuCl}_{2} \text {, }
$$

seine Anionen haben also die Formel: $\mathrm{CuCl}_{2}{ }^{\prime}$.

Würden wir nicht die Anzahl der Kupferatome in dem komplexen Salz von den früheren Messungen kennen, so wäre die elektromotorische Kraft dieser Kette auch vereinbar mit der Annahme, dafs das komplexe Salz die Formel:

$$
\mathrm{K}_{2} \mathrm{Cu}_{2} \mathrm{Cl}_{4} \text { oder } \mathrm{K}_{3} \mathrm{Cu}_{3} \mathrm{Cl}_{8} \text { hätte. }
$$


Die folgende 'Tabelle ergiebt in der ersten Spalte die Konzentrationen des Kupfers, in den beiden folgenden die des Chlorkaliums in Normalitäten, in der vierten und fünften das Verhältnis beider Konzentrationen, in der sechsten die für die ursprüngliche Potentialdifferenz der Metalle korrigierten elektromotorischen Kräfte, in der siebenten und achten den Werth $\left(\frac{m+n}{n}\right)$ bezw. $\left(\frac{m+n}{n}\right)^{\prime}$

Tabelle 9.

Konzentrationsketten mit gleichem Kupfer- und wechselndem Chlorgehalt.

\begin{tabular}{|c|c|c|c|c|c|c|c|}
\hline \multirow{2}{*}{$\begin{array}{c}\text { Kupfergehalt } \\
\text { g-Atome }\end{array}$} & \multicolumn{2}{|c|}{$\begin{array}{c}\text { Chlorkalium- } \\
\text { gehalt } \\
\text { g-Moleküle }\end{array}$} & \multicolumn{2}{|c|}{$\begin{array}{l}\text { Chorionen- } \\
\text { Verhältnis }\end{array}$} & \multirow{2}{*}{$\begin{array}{c}E^{\prime} \\
\text { Volt }\end{array}$} & \multirow[t]{2}{*}{$\left(\frac{m+n}{n}\right)$} & \multirow[t]{2}{*}{$\left(\frac{m+n}{n}\right)^{\prime}$} \\
\hline & $(\mathrm{KCl})_{1}$ & (KCl) & $(\mathrm{Cl})_{2}{ }^{\prime}:(\mathrm{Cl})^{\prime}$ & $\left.(\mathrm{Cl})_{1}{ }^{\prime \prime}\right)(\mathrm{Cl})^{\prime \prime}$ & & & \\
\hline 0.00008 & 0.2 & 0.05 & 8.73 & 3.73 & 0.0680 & 2.05 & 2.05 \\
\hline 0.0002 & 0.22 & 0.1 & 2.1 & 2.1 & 0.0374 & 2.00 & 2.00 \\
\hline 0.00014 & 0.3 & 0.1 & 2.8 & 2.8 & 0.0524 & 2.01 & 2.01 \\
\hline 0.00007 & 0.4 & 0.1 & 3.69 & 3.69 & 0.0734 & 2.23 & 2.23 \\
\hline 0.05 & 2.0 & 0.642 & 3.0 & 3.2 & 0.0805 & 2.90 & 2.75 \\
\hline 0.0425 & 2.0 & 0.66 & 2.87 & 3.02 & 0.0803 & 3.03 & 2.89 \\
\hline
\end{tabular}

Die Messung der elektromotorischen Kräfte und Ketten der zuerst beschriebenen Art hatte ergeben, dals auch in den konzentrierteren Chlorkaliumlösungen die konplexen Ionen nur je ein Atom Kupfer enthalten. Für die Berechnung des Verhältnisses der Chlorionen ist es wichtig, zu wissen, wie viel von dem Chlorkalium unter Bildung des Komplexsalzes gebunden wird; um nicht das, was zu beweisen ist, schon als bewiesen annehmen zu müssen, wurde die Berechnung so geführt, dafs für jedes Kupferatom der Lösung ein oder zwei Moleküle des Chlorkaliums als gebunden angesehen wurden.

Die Spalten vier und fünf, bezw. sieben und acht zeigen, dals sich daraus für die verdünnten Chlorkaliumlösungen kein Unterschied im Resultat ergiebt; aber auch für die konzentrierteren Lösungen ist der Unterschied ziemlich klein und nicht geeignet, das Gesamtergebnis zu modifizieren.

Es zeigt sich also, dafs auf ein Atom Kupfer in den verdünnteren Chlorkaliumlösungen zwei Atome, in den konzentrierteren 
drei Atome Chlor kamen. In den verdünnteren Lösungen enthalten die Moleküle - wie die früheren Versuche gezeigt haben nur ein Atom Kupfer, die Formel des komplexen Salzes ist also: $\mathrm{KCuCl}_{2}$.

Auffällig könnte es erscheinen, dalis auch die 0.05 normale Chlorkaliumlösung gegen die 0.2 normale nahezu die berechnete elektromotorische Kraft giebt, wie wohl doch in ihr neben den komplexen Ionen auch Ionen des Cuprisalzes vorhunden sind.

Die Störung, welche durch diesen Umstand bei den Versuchen mit gleichbleibender Chlorkonzentration beobachtet wurde, fällt aber hier fort, weil in Berührung mit dem Kupfer in der konzentrierten Chlorkaliumlösung das Cuprichlorid zu Cuprochlorid reduziert wird, welches als komplexes Salz in Lösung geht. Dafür spricht, dafs die elektromotorische Kraft dieser Kette beim schnellen Schütteln dieser Lösung um 5-8 Millivolt zurückging, dafs aber der normale Wert sich sofort einstellte, wenn die Lösung auch nur eine Minute wieder in Ruhe sich befindet. So lange geschüttelt wird, kommt immer eine an Cupriionen reichere Lösung in der konzentrierteren Chlorkaliumlösung mit der Kupferelektrode in Berührung, sowie die Lösung steht, werden Cupriionen aus der unmittelbaren Nachbarschaft der Elektrode durch das Kupfer reduziert, da sie in der konzentrierten Chlorkaliumlösung neben Kupfer nicht existenzfähig sind; dadurch wird beim Stehen die Menge der freien Cuproionen in der konzentrierteren Lösung vermindert und die elektromotorische Kraft steigt. Die Formel der in den für Chlorkalium stärker als 0.05 normalen Lösungen enthaltenen Komplexionen mufs $\mathrm{Cu}_{n} \mathrm{Cl}_{3 n}$ sein, wobei $n$ den Wert eins, zwei u. s. w. haben könnte. Aus der oben angeführten Messung der elektromotorischen Kraft bei verschiedenem Kupfergehalt in beiden Lösungen ergiebt sich, dafs $n$ den Wert 1 besitzt, dals also die Formel der komplexen Ionen $\mathrm{CuCl}_{3}{ }^{\prime \prime}$, die des komplexen Kaliumsalzes $\mathrm{K}_{2} \mathrm{CuCl}_{3}$ ist.

Die Grenze zwischen den Chlorkaliumkonzentrationen, in denen die Komplexionen die Formel $\mathrm{CuCl}_{2}{ }^{\prime}$ und $\mathrm{CuCl}_{3}{ }^{\prime \prime}$ haben, ist natürlich nicht scharf, es ergiebt sich aus der Tabelle 9 , dafs in 0.4 normalen Lösungen schon Ionen $\mathrm{CuCl}_{3}{ }^{\prime \prime}$ neben den überwiegenden Ionen $\mathrm{CuCl}_{2}{ }^{\prime}$ vorhanden sind.

Es ist hierdurch nachgewiesen, dafs die Annahme von ABEL, wonach die konzentrierteren Lösungen Ionen der Formel $\mathrm{Cu}_{2} \mathrm{Cl}_{4}$ " ent- 
halten, irrig ist. $\mathrm{ABEu}^{1}$ und Hausnath ${ }^{2}$ haben für diese Formel auch darin eine Stütze zu finden geglaubt, dafs der Gefrierpunkt normaler Salzsäure auf Zusatz ron Cuprochlorid steigt; eine Verminderung der Zahl der freien Moleküle und lonen ist dadurch sicher bewiesen; eine solche tritt aber nicht nur ein, wenn durch Zufügung von Cuprochlorid aus zwei Chlorionen ein Ion $\mathrm{Cu}_{2} \mathrm{Cl}_{4}$ entsteht, was ABEI annimmt, sondern auch, wenn aus zwei Chlorionen ein Ion $\mathrm{CuCl}_{3}$ entsteht. Nur mürste im ersteren Falle die Gefrierpunktserhöhung für jedes Atom Kupfer halb so grofs sein, als im zweiten Falle, wie die Gleichungen:

$$
\begin{aligned}
& \mathrm{Cu}_{2} \mathrm{Cl}_{2}+2 \mathrm{Cl}^{\prime}=\mathrm{Cu}_{2} \mathrm{Cl}_{4}{ }^{\prime \prime} \\
& \mathrm{CuCl}+2 \mathrm{Cl}^{\prime}=\mathrm{CuCl}_{3}{ }^{\prime \prime}
\end{aligned}
$$

ergeben. Abel und HausRath beobachteten in der That eine gröfsere Gefrierpunktserhöhung, als nach der von ihnen angenommenen ersten Gleichung zu erwarten war. Eine sichere Entscheidung konnte aber nur die Messung der Konzentrationskette ergeben.

Die Ergebnisse vorstehender Arbeit lassen sich in Kürze wie folgt zusammenfassen:

1. Cuprochlorid erleidet durch Wasser eine Spaltung einerseits in Kupferhydroxydul und freie Salzsäure, anderseits und überwiegend in Cuprichlorid und metallisches Kupfer.

2. Die letztere Spaltung wird durch Zusatz steigender Mengen von Chloriden zurückgedrängt, so dafs in Lösungen, die stärker als 0.05 normal für Chlorkalium sind, das Cuprochlorid sich ohne Zersetzung löst.

3. Es wurde die Löslichkeit des Cuprochlorids in reinem Wasser und in Chlorkaliumlösungen bestimmt.

4. In den Lösungen in Wasser ist das Cuprochlorid zum Teil in freiem Zustande bezw. als dessen freie Ionen, zum Teil in Form komplexer Ionen enthalten.

5. Es wurde eine auf der Messung von Konzentrationsketten beruhende Methode zur Bestimmung der Formel, welche komplexe Salze in Lösung haben, erprobt.

1. e. S. 405 .

2 Inaugural-Dissertation Göttingen 1901. 
6. Lösungen von 0.05-0.4 normal Chlorkalium lösen Cuprochlorid unter Bildung der Verbindung $\mathrm{KCuCl}_{2}$ auf, während in Chloridlösungen von höherer Konzentration die Formel des komplexen Salzes $\mathrm{K}_{2} \mathrm{CuCl}_{3}$ ist.

7. Während die Moleküle der komplexen Cuprosalze sicher nur ein Atom Kupfer enthalten, konnte noch nicht endgültig entschieden werden, ob die freien Cuproionen ein- oder zweiatomig sind.

Braunschweig, Elektrochemisches Laboratorium der Techn. Hochschule.

Bei der Redaktion eingegangen am 8. Januar 1902. 